\title{
WIENER'S CRITERION FOR PARABOLIC EQUATIONS WITH VARIABLE COEFFICIENTS AND ITS CONSEQUENCES
}

\author{
NICOLA GAROFALO AND ERMANNO LANCONELLI
}

Dedicated to Bruno Pini

\begin{abstract}
In a bounded set in $\mathbf{R}^{n+1}$ we study the problem of the regularity of boundary points for the Dirichlet problem for a parabolic operator with smooth coefficients. We give a geometric characterization, modelled on Wiener's criterion for Laplace's equation, of those boundary points that are regular. We also present some important consequences. Here is the main one: a point is regular for a variable coefficient operator if and only if it is regular for the constant coefficient operator obtained by freezing the coefficients at that point.
\end{abstract}

1. Introduction and statement of the results. A basic issue in potential theory is the convergence of the solution of the Dirichlet problem to the prescribed boundary value. The primary motivation of this paper is to investigate such a question for parabolic operators with smooth coefficients. As a result, given a bounded open subset $D \subset \mathbf{R}^{n+1}$, we obtain a geometric characterization of those points $\left(x_{0}, t_{0}\right) \in \partial D$ which are regular for the Dirichlet problem.

In $\mathbf{R}^{n+1}$ we consider the second order equation

$$
L u=\operatorname{div}(A(x, t) \nabla u)-D_{t} u=0,
$$

where $A(x, t)=\left(a_{i j}(x, t)\right), i, j=1, \ldots, n$, is a real, symmetric, matrix-valued function on $\mathbf{R}^{n+1}$ with $C^{\infty}$ entries. We assume that there exists $\nu \in(0,1]$ such that for every $\xi \in \mathbf{R}^{n}$ and every $(x, t) \in \mathbf{R}^{n+1}$,

$$
\nu|\xi|^{2} \leq \sum_{i, j=1}^{n} a_{i j}(x, t) \xi_{i} \xi_{j} \leq \nu^{-1}|\xi|^{2} .
$$

As the results in this paper are of a local nature we also make the nonrestrictive assumption that there exists a compact set $F_{0} \subset \mathbf{R}^{n+1}$, containing the origin, such that for $i, j=1, \ldots, n$

$$
a_{i j}(x, t)=\delta_{i j} \quad \text { for }(x, t) \in \mathbf{R}^{n+1} \backslash F_{0} .
$$

A bounded open set $U \subset \mathbf{R}^{n+1}$ is said to be $L$-regular if for any $\varphi \in C(\partial U)$ there exists a (unique) $H_{\varphi}^{U} \in C^{\infty}(U) \cap C(U)$ such that $L H_{\varphi}^{U}=0$ in $U$, and for which for every $\left(x_{0}, t_{0}\right) \in \partial U$

$$
\lim _{\substack{(x, t) \rightarrow\left(x_{0}, t_{0}\right) \\(x, t) \in U}} H_{\varphi}^{U}(x, t)=\varphi\left(x_{0}, t_{0}\right) .
$$

Received by the editors June 9, 1987 .

1980 Mathematics Subject Classification (1985 Revision). Primary 31B10, 31B20, 35K20.

Key words and phrases. Parabolic equations, Wiener's criterion, mean value properties. 
A function $w: D \rightarrow \overline{\mathbf{R}}$ is said L-superparabolic in $D$ if: (i) $-\infty<w \leq+\infty$, $w<+\infty$ in a dense subset of $D$; (ii) $w$ is lower semicontinuous (l.s.c.); (iii) for every $L$-regular subset $U \subset \bar{U} \subset D$, and every $\varphi \in C(\partial U)$, if $w \mid \partial U \geq \varphi$, then $w \geq H_{\varphi}^{U}$ in $U$.

A function $w: D \rightarrow \overline{\mathbf{R}}$ is said to be $L$-subparabolic in $D$ if $-w$ is $L$-superparabolic. We say that $w$ is $L$-parabolic if $w$ is both $L$-super and $L$-subparabolic.

For an arbitrary bounded open subset $D \subset \mathbf{R}^{n+1}$, and an arbitrary $\varphi \in C(\partial D)$, a generalized solution, $u$, of the Dirichlet problem

$$
\left\{\begin{array}{l}
L u=0 \text { in } D \\
\left.u\right|_{\partial D}=\varphi
\end{array}\right.
$$

is provided by the Perron-Wiener-Brelot-Bauer method as

$$
u=\inf \{w \mid w \text { is } L \text {-superparabolic in } D \text { and } \liminf w \geq \varphi \text { on } \partial D\} .
$$

Classical potential theory assures that $u \in C^{\infty}(D)$ and that $L u=0$ in the classical sense. However, it is not true in general that $u$ attains continuously the boundary value $\varphi$. A point $\left(x_{0}, t_{0}\right) \in \partial D$ is said to be $L$-regular if

$$
\lim _{(x, t) \rightarrow\left(x_{0}, t_{0}\right)} u(x, t)=\varphi\left(x_{0}, t_{0}\right)
$$

for any $\varphi \in C(\partial D)$, where $u$ is defined as in (1.5). In order to state our results we need to introduce a few more definitions. We let $\Gamma(x, t ; y, s)$ denote the fundamental solution of $L$ in (1.1). By virtue of the assumptions made on the matrix of the coefficients, $\Gamma(x, t ; y, s)$ is a $C^{\infty}$ function for $(x, t) \neq(y, s)$. We denote by $M^{+}\left(\mathbf{R}^{n+1}\right)$ the set of all nonnegative Radon measures on $\mathbf{R}^{n+1}$. If $F \subseteq \mathbf{R}^{n+1}$ is closed we let $M^{+}(F)=\left\{\mu \in M^{+}\left(\mathbf{R}^{n+1}\right) \mid \operatorname{supp} \mu \subseteq F\right\}$. For $\mu \in M^{+}\left(\mathbf{R}^{n+1}\right)$ the function

$$
\Gamma_{\mu}(x, t)=\int_{\mathbf{R}^{n+1}} \Gamma(x, t ; y, s) d \mu(y, s)
$$

is called the $L$-potential of $\mu$. For a closed set $F \subset \mathbf{R}^{n+1}$ the $L$-capacity of $F$ is defined as

$$
\operatorname{cap}_{L}(F)=\sup \left\{\mu\left(\mathbf{R}^{n+1}\right) \mid \mu \in M^{+}(F), \Gamma_{\mu} \leq 1 \text { on } \mathbf{R}^{n+1}\right\} .
$$

Finally, given $\left(x_{0}, t_{0}\right) \in \mathbf{R}^{n+1}$ and a $k \in \mathbf{N}$ we define for $\lambda \in(0,1)$

$$
\begin{aligned}
A\left(x_{0}, t_{0} ; \lambda^{k}\right)= & \left\{(x, t) \in \mathbf{R}^{n+1} \mid\left(4 \pi \lambda^{k+1}\right)^{-n / 2} \geq \Gamma\left(x_{0}, t_{0} ; x, t\right) \geq\left(4 \pi \lambda^{k}\right)^{-n / 2}\right\} \\
& \cup\left\{\left(x_{0}, t_{0}\right)\right\} .
\end{aligned}
$$

$A\left(x_{0}, t_{0} ; \lambda^{k}\right)$ is the "annular" region between the two level sets $\left\{\Gamma\left(x_{0}, t_{0} ; x, t\right)=\right.$ $\left.\left(4 \pi \lambda^{k+1}\right)^{-n / 2}\right\}$ and $\left\{\Gamma\left(x_{0}, t_{0} ; x, t\right)=\left(4 \pi \lambda^{k}\right)^{-n / 2}\right\}$ of the fundamental solution $\Gamma$.

One of the main results in this paper is the following

THEOREM 1.1. Given a bounded open subset $D \subset \mathbf{R}^{n+1}$, a point $\left(x_{0}, t_{0}\right) \in \partial D$ is $L$-regular iff for every $\lambda \in(0,1)$

$$
\sum_{k=1}^{\infty} \lambda^{-k n / 2} \operatorname{cap}_{L}\left(D^{c} \cap A\left(x_{0}, t_{0} ; \lambda^{k}\right)\right)=+\infty
$$

where $D^{c}=\mathbf{R}^{n+1} \backslash D$. 
We remark explicitly that the behavior of the series in (1.10) does not depend on $\lambda \in(0,1)$.

In order to state the next theorem we consider another second order operator in $\mathbf{R}^{n+1}$

$$
M=\operatorname{div}(B(x, t) \nabla)-D_{t} .
$$

$B(x, t)=\left(b_{i j}(x, t)\right), i, j=1, \ldots, n$, is a real, symmetric, matrix-valued function on $\mathbf{R}^{n+1}$ with $C^{\infty}$ entries, for which there exists a $\mu \in(0,1]$ such that

$$
\mu|\xi|^{2} \leq \sum_{i, j=1}^{n} b_{i j}(x, t) \xi_{i} \xi_{j} \leq \mu^{-1}|\xi|^{2}
$$

for every $\xi \in \mathbf{R}^{n}$ and every $(x, t) \in \mathbf{R}^{n+1}$.

The second main result in this paper is the following

THEOREM 1.2. Let $L$ and $M$ be two parabolic operators as in (1.1) and (1.11), for which (1.2) and (1.12) hold respectively. Let $D$ be a bounded open subset of $\mathbf{R}^{n+1}$, and let $\left(x_{0}, t_{0}\right) \in \partial D$ be such that

$$
A\left(x_{0}, t_{0}\right)=B\left(x_{0}, t_{0}\right) .
$$

Then $\left(x_{0}, t_{0}\right)$ is L-regular iff it is $M$-regular.

As a consequence of Theorem 1.2 we obtain

COROLlaRY 1.1. Let $L$ be as in (1.1) and let $D$ be a bounded open subset of $\mathbf{R}^{n+1}$. Then $\left(x_{0}, t_{0}\right) \in \partial D$ is $L$-regular iff $\left(x_{0}, t_{0}\right)$ is $L_{0}$-regular, where $L_{0}$ is the constant coefficient operator

$$
L_{0}=\operatorname{div}\left(A\left(x_{0}, t_{0}\right) \nabla\right)-D_{t},
$$

obtained from $L$ by freezing the coefficients at $\left(x_{0}, t_{0}\right)$.

The proofs of Theorems 1.1 and 1.2 will be given in $\S 4$ of this paper. $\S \S 2$ and 3 are devoted to establishing several results which constitute the building blocks of $\S 4$. Before describing the plan of the paper we give a brief discussion of the historical background.

Theorem 1.1 is modelled on Wiener's well-known criterion for the regularity of a boundary point for Laplace's operator. In 1924 Wiener [W] proved that: given a bounded domain $\Omega \subset \mathbf{R}^{n}$, a point $x_{0} \in \partial \Omega$ is $\Delta$-regular iff for every $\lambda>1$

$$
\sum_{k=1}^{\infty} \lambda^{k} \operatorname{cap}_{\Delta}\left(\Omega^{c} \cap\left\{x \in \mathbf{R}^{n} \mid \lambda^{k+1} \geq G\left(x_{0}-x\right) \geq \lambda^{k}\right\}\right)=+\infty .
$$

In (1.15) $\operatorname{cap}_{\Delta}(\cdot)$ denotes the Newtonian capacity, $\Omega^{c}=\mathbf{R}^{n} \backslash \Omega$, and $G\left(x_{0}-x\right)$ the fundamental solution of $\Delta$ with pole at $x=x_{0}$. In 1982 Evans and Gariepy succeeded in extending Wiener's criterion to the heat operator $H=\Delta-D_{t}$ in $\mathbf{R}^{n+1}$. They proved (see $[\mathbf{E G}]$ )

THEOREM (EVANS AND GARIEPY). Let $D$ be a bounded open subset of $\mathbf{R}^{n+\mathbf{i}}$. $A$ point $\left(x_{0}, t_{0}\right) \in \partial D$ is $H$-regular iff for every $\lambda \in(0,1)$

$$
\sum_{k=1}^{\infty} \lambda^{-k n / 2} \operatorname{cap}_{H}\left(D^{c} \cap A\left(x_{0}, t_{0} ; \lambda^{k}\right)\right)=+\infty .
$$


In (1.16) $\operatorname{cap}_{H}(\cdot)$ denotes the heat-capacity defined as in (1.8) using the GaussWeierstrass kernel

$$
\begin{aligned}
K(x, t ; y, s) & =K(x-y ; t-s) \\
& = \begin{cases}(4 \pi(t-s))^{-n / 2} \exp \left(-\frac{|x-y|^{2}}{4(t-s)}\right), & t>s, \\
0, & t \leq s,\end{cases}
\end{aligned}
$$

whereas $A\left(x_{0}, t_{0} ; \lambda^{k}\right)$ is defined as in (1.9), using $K$ instead of $\Gamma$. One should mention that the "only if" part of the previous theorem had already been proved by one of the authors in [L1]. Theorem 1.1 above extends Evans and Gariepy's result to parabolic operators with smooth variable coefficients.

The difficulties one encounters in passing from elliptic to parabolic equations account for the time-lag between Wiener's and Evans and Gariepy's results. These difficulties are due to the evolutive nature of the operator $H$, a fact which is reflected in the form of the fundamental solution $K$ in (1.17). The ingenious strategy in [EG] is based on two main ingredients:

(1) Representation formulas for solutions of $H u=0$ as averages on the level sets of the fundamental solution $K$; (2) A strong form of the Harnack inequality controlling a positive solution of $H u=0$ in suitable regions exterior to the parabolic ones in which the usual Harnack inequality holds. Our proof of Theorem 1.1 follows the general lines of Evans and Gariepy's proof for the constant coefficients case. However, most of the proofs in [EG] relied on the explicit knowledge of the fundamental solution $K$ in (1.17) of the heat operator. For an operator with variable coefficients the fundamental solution is not known explicitly, a fact which introduces a new order of difficulty in the problem. The ad hoc tool to overcome this difficulty is provided by the following asymptotic estimate for the proof of which we refer to $[\mathbf{G L}]$. For related results we refer the reader to $[\mathbf{B G M}]$ and $[\mathbf{K}]$.

THEOREM 1.3. Let $K$ be a sufficiently small compact neighborhood of the origin in $\mathbf{R}^{n}$. For $x, y \in K, x \neq y$, let $\Gamma(x, y, t)=\Gamma(x, t ; y, 0)$ denote the fundamental solution of $L$ in $(1.1)$ with pole at $(y, 0)$. Then as $t \rightarrow 0^{+}$we have the asymptotic expansion,

$$
\Gamma(x, y, t) \sim(4 \pi t)^{-n / 2} \exp \left(-\frac{d^{2}(x, y, t)}{4 t}\right) \sum_{j=0}^{\infty} t^{j} u_{j}(x, y, t),
$$

where, for every $t \geq 0, d(x, y, t)$ denotes the distance from $x$ to $y$ in the Riemannian metric on $K, d s_{t}^{2}=g_{i j}(t) d x_{i} \otimes d x_{j}, g_{i j}(t)(x)=\left(a^{i j}(x, t)\right)$, if $\left(a^{i j}(x, t)\right)=A^{-1}(x, t)$. The function $u_{0}$ in the expansion (1.18) can be chosen such that $u_{0}(x, x, 0)=1$. $B y(1.18)$ we mean that there exist a suitably small $T>0$ and a sequence $\left(u_{j}\right)_{j \in \mathbf{N}}$, with $u_{j} \in C^{\infty}(K \times K \times[0, T])$, such that for every $k \in \mathbf{N} \cup\{0\}$ we can find $w_{k}: K \times K \times(0, T) \rightarrow \mathbf{R}$ such that

$$
\Gamma(x, y, t)-(4 \pi t)^{-n / 2} \exp \left(-\frac{d^{2}(x, y, t)}{4 t}\right) \sum_{j=0}^{k} t^{j} u_{j}(x, y, t)=w_{k}(x, y, t)
$$

with

$$
w_{k}(x, y, t)=O\left(t^{k-n / 2} \exp \left(-\frac{\delta|x-t|^{2}}{4 t}\right)\right) \quad \text { as } t \rightarrow 0^{+}
$$


uniformly for $x, y \in K$. In (1.20) $\delta>0$ is a number depending on $\nu$ in (1.2) and $n$. An expansion similar to (1.18) holds for the derivatives of $\Gamma$.

Theorem 1.3 plays a crucial role in this paper. With it in hand we have been able to prove a strong form of Harnack's inequality which provides quantitative information on a positive solution, $u$, of (1.1) in regions exterior to those in which the usual Harnack inequality holds. In order to state this result we introduce some notation. For $z_{0}=\left(x_{0}, t_{0}\right), z=(x, t) \in \mathbf{R}^{n+1}$ and $r>0$ we set

$$
\begin{aligned}
& \Psi_{r}\left(z_{0}\right)=\left\{z \in \mathbf{R}^{n+1} \mid \Gamma\left(z_{0} ; z\right)=(4 \pi r)^{-n / 2}\right\} \cup\left\{z_{0}\right\}, \\
& \Omega_{r}\left(z_{0}\right)=\left\{z \in \mathbf{R}^{n+1} \mid \Gamma\left(z_{0} ; z\right)>(4 \pi r)^{-n / 2}\right\} .
\end{aligned}
$$

We call $\Psi_{r}\left(z_{0}\right)$ and $\Omega_{r}\left(z_{0}\right)$ respectively the parabolic sphere and the parabolic ball of radius $r$ and center at $z_{0}$.

$$
\Psi_{r}^{*}\left(z_{0}\right)=\left\{z \in \mathbf{R}^{n+1} \mid \Gamma\left(z ; z_{0}\right)=(4 \pi r)^{-n / 2}\right\} \cup\left\{z_{0}\right\},
$$

and

$$
\Omega_{r}^{*}\left(z_{0}\right)=\left\{z \in \mathbf{R}^{n+1} \mid \Gamma\left(z ; z_{0}\right)>(4 \pi r)^{-n / 2}\right\},
$$

are called respectively the adjoint parabolic sphere, and the adjoint parabolic ball with radius $r$ and center at $z_{0}$. In the sequel, see $\S 2$ below, $\alpha_{1}, \eta, \sigma, \eta_{1}$ will be numbers fixed throughout the discussion which solely depend on the eigenvalue $\nu$ in (1.2) and on the compact $F_{0}$ in (1.3). For $t<0$ and $r>0$ we set

$$
\begin{aligned}
R_{r}^{1}(t) & =2 n \alpha_{1}(-t) \ln \left(-r \eta_{1} / t\right), \\
Q_{r} & =\left\{z \in \Omega_{r} \mid t>-\eta r / 2\right\},
\end{aligned}
$$

where in (1.26) $\Omega_{r}=\Omega_{r}(0,0)$. One should keep in mind that $\sigma$ and $\eta$ have been chosen in such a way that $\Omega_{\sigma r} \subset Q_{2 r}$, for every $r>0$, and moreover $-\eta r<$ $\inf \left\{t \mid(x, t) \in \Omega_{\sigma r}\right\}$ (see §2). Therefore, if we let

$$
I_{r}=\left\{\left.z \in \mathbf{R}^{n+1}|| x\right|^{2} \leq R_{r}^{1}(-\eta r), t=-\eta r\right\},
$$

the two sets $I_{r}$ and $Q_{\sigma r}$ are detached and there is a time-lag between them. Since for $\rho<r \Omega_{\rho} \subset \Omega_{r}$, this is true also for $I_{r}$ and $\Omega_{(3 / 4) \sigma r}$. We can now state the following

THEOREM 1.4 (STRONG HARNACK INEQUALITY). There exist $r_{0}>0$ and $a$ $\Lambda>0$ such that if $u>0$ is a solution of $L u=0$ in $Q_{2 r}$, with $u \in C\left(\partial Q_{2 r} \backslash\{(0,0)\}\right)$, then

$$
f_{I_{r}} u(x,-\eta r) d x \leq \Lambda \inf _{\Omega_{(3 / 4) \sigma r}} u
$$

for every $r \leq r_{0} . \Lambda$ in (1.28) does not depend on $u$.

In (1.28) $f_{I_{r}}$ denotes the $n$-dimensional average on $I_{r}$. We remark that Theorem 1.4 cannot be obtained just from the usual Harnack inequality since the set $\Omega_{(3 / 4) \sigma r}$ includes a region which is exterior to any paraboloid $\left\{t=-\delta r|x|^{2}\right\}$ with vertex at $(0,0)$ and opening in the negative time-direction. Theorem 1.4 extends to our setting Lemma 3.2 in [EG]. The main tool in the proof of (1.28) is the construction of a suitable subsolution of $L$ which vanishes on the level set $\Psi_{\sigma r}$ of the fundamental 
solution $\Gamma$. We accomplish this by choosing a suitable concave function $\psi: \mathbf{R} \rightarrow \mathbf{R}$ and setting

$$
v(x, t)=\psi\left(\ln \left[(4 \pi \sigma r)^{n / 2} \Gamma(0,0 ; x, t)\right]\right)
$$

(see $\S 2$ below). The core of the proof of Theorem 1.4 is to show that $L v \geq 0$ in that part of the parabolic ball $\Omega_{\sigma r}$ which lies outside a paraboloid. To prove this we must use the full strength of Theorem 1.3.

$\S 3$ is dedicated to establish some mean-value formulas for solutions of $L u=f$ on the sets $\Psi_{r}$ and $\Omega_{r}$ introduced in (1.21), (1.22). In what follows we indicate by the letters $z, z_{0}, \varsigma$ the points in $\mathbf{R}^{n+1},(x, t),\left(x_{0}, t_{0}\right),(\xi, \tau)$ respectively. We will prove the following

THEOREM 1.5. Let $u \in C^{\infty}\left(\mathbf{R}^{n+1}\right)$ and let $z_{0} \in \mathbf{R}^{n+1}$. For a.e. $r>0$ we have

$$
\begin{aligned}
& -\int_{\Psi_{r}\left(z_{0}\right)} u(\varsigma) A(\varsigma)\left(\nabla_{\zeta} \Gamma\left(z_{0} ; \zeta\right)\right) \cdot \vec{N}_{\xi}(\varsigma) d H_{n}(\varsigma) \\
& \quad=u\left(z_{0}\right)+\int_{\Omega_{r}\left(z_{0}\right)} L u(z)\left[\Gamma\left(z_{0} ; z\right)-(4 \pi r)^{-n / 2}\right] d z .
\end{aligned}
$$

For every $r>0$ we have

$$
\begin{aligned}
& (4 \pi r)^{-n / 2} \int_{\Omega_{r}\left(z_{0}\right)} u(z) \frac{A(z)\left(\nabla_{x} \Gamma\left(z_{0} ; z\right)\right) \cdot \nabla_{x} \Gamma\left(z_{0} ; z\right)}{\Gamma^{2}\left(z_{0} ; z\right)} d z \\
& \quad=u\left(z_{0}\right)+\frac{n}{2} r^{-n / 2} \int_{0}^{r} l^{n / 2} \int_{\Omega_{l}\left(z_{0}\right)} L u(z)\left[\Gamma\left(z_{0} ; z\right)-(4 \pi l)^{-n / 2}\right] d z \frac{d l}{l} .
\end{aligned}
$$

In (1.30) $\vec{N}_{\xi}$ denotes the spatial component of the outer normal $\vec{N}=\left(\vec{N}_{\xi}, N_{\tau}\right)$ to the surface $\Psi_{r}\left(z_{0}\right)$.

Theorem 1.5 generalizes mean-value formulas for solutions of $L u=0$ found by E. Fabes and one of the authors in [FG]. In fact, if in (1.30), (1.31) $u$ is a solution of $L u=0$, Theorem 1.5 gives back Theorems 1 and 2 in [FG]. In particular, if $L=H$, so that $\Gamma=K$ (see (1.17)), and $u$ is a solution of $H u=0$, we obtain from (1.31)

$$
u\left(z_{0}\right)=(4 \pi r)^{-n / 2} \int_{\Omega_{r}\left(z_{0}\right)} u(z) \frac{\left|x_{0}-x\right|^{2}}{4\left(t_{0}-t\right)^{2}} d z .
$$

Mean-value formulas for temperatures in $\mathbf{R}^{n+1}$ (solutions of $H u=0$ ) have a long history. Pini was the first one to prove and use a surface mean-value formula for temperatures in $\mathbf{R}^{2}$ (see [P1, P2, P3]). Subsequently, Fulks extended Pini's result to temperatures in $\mathbf{R}^{n+1}$ [Fu]. Finally, Watson proved (1.32) starting from Fulks result [Wa1]. Evans and Gariepy made the key observation that (1.32) could be used to estimate from above the capacity of the "annular" regions $A\left(x_{0}, t_{0} ; \lambda^{K}\right)$ in (1.16). This is done through the following lemma and its corollary. In the sequel, with $K$ as in (1.17), we will denote $\Omega_{r}=\left\{z \in \mathbf{R}^{n+1} \mid K(-z)>(4 \pi r)^{-n / 2}\right\}$ (see (1.22)).

Lemma (SEE $[\mathbf{E G}])$. For $u \in C^{\infty}\left(\mathbf{R}^{n+1}\right)$ let

$$
\Phi(r)=r^{-n / 2} \int_{\Omega_{r}} u(z) \frac{|x|^{2}}{t^{2}} d z .
$$


Then

$$
\Phi^{\prime}(r)=\frac{n}{2} r^{-(n+2) / 2} \int_{\Omega_{r}} H u(z)\left(\frac{R_{r}(t)-|x|^{2}}{t}\right) d z,
$$

where for $t<0, R_{r}(t)=2 n(-t) \ln (-r / t)$.

COROLlaRY (SEE [EG]). Suppose that $H u \geq 0$ in $\Omega_{2 r}$. Then there exists $C_{n}>0$ such that

$$
\Phi(2 r)-\Phi(r) \geq C_{n} r^{-n / 2} \int_{\Omega_{r / 2}} H u(z) d z .
$$

In [EG] (1.34) is proved by a direct computation similar to that which leads to Rellich's identity for the Laplacian. This is possible because of the explicit knowledge of the fundamental solution $K$ of $H$. In our case this approach does not work. However, if we set for $z_{0} \in \mathbf{R}^{n+1}$ and $r>0$

$$
u_{r}\left(z_{0}\right)=(4 \pi r)^{-n / 2} \int_{\Omega_{r}\left(z_{0}\right)} u(z) \frac{A(z)\left(\nabla_{x} \Gamma\left(z_{0} ; z\right)\right) \cdot \nabla_{x} \Gamma\left(z_{0} ; z\right)}{\Gamma^{2}\left(z_{0} ; z\right)} d z,
$$

(1.31) in Theorem 1.5 provides an expression for $u_{r}\left(z_{0}\right)$. Using it we prove

THEOREM 1.6. Let $u \in C^{\infty}\left(\mathbf{R}^{n+1}\right), z_{0} \in \mathbf{R}^{n+1}$, and let $u_{r}\left(z_{0}\right)$ be defined as in (1.36). Then

$$
\frac{d}{d r} u_{r}\left(z_{0}\right)=\frac{n}{2 r}\left(\frac{1}{4 \pi r}\right)^{n / 2} \int_{\Omega_{r}\left(z_{0}\right)} L u(z) \ln \left[(4 \pi r)^{n / 2} \Gamma\left(z_{0} ; z\right)\right] d z .
$$

COROLlaRY 1.2. For $z_{0} \in \mathbf{R}^{n+1}$ and $\alpha>1$ let $u \in C^{\infty}\left(\Omega_{\alpha^{2} r}\left(z_{0}\right)\right)$, with $L u \geq 0$ in $\Omega_{\alpha^{2} r}\left(z_{0}\right)$. Then there exists a positive constant $C=C(n, \alpha)$ such that

$$
u_{\alpha^{2} r}\left(z_{0}\right)-u_{\alpha r}\left(z_{0}\right) \geq C r^{-n / 2} \int_{\Omega_{r}\left(z_{0}\right)} L u(z) d z .
$$

REMARK. If in Theorem 1.6 $L=H$, so that $\Gamma=K$, we obtain

$$
\ln \left[(4 \pi r)^{n / 2} K\left(z_{0}-z\right)\right]=\frac{R_{r}\left(t_{0}-t\right)^{2}-\left|x_{0}-x\right|^{2}}{4\left(t_{0}-t\right)} .
$$

Therefore, (1.34) is a special case of (1.37).

Theorem 1.3 and Corollary 1.2 are the primary ingredients in the proof of Theorem 1.1. However, there is still a piece of information missing. In the course of the proof of the sufficiency of $(1.10)$ it is crucial at one point to be able to deal with regularization of equilibrium potentials, rather than with the potentials themselves. Since usual mollification does not work in that context our idea has been to use the averaging operator $u \rightarrow u_{r}, r>0$, where for $z \in \mathbf{R}^{n+1}$

$$
u_{r}(z)=(4 \pi r)^{-n / 2} \int_{\Omega_{r}(z)} u(\varsigma) \frac{A(\varsigma)\left(\nabla_{\xi} \Gamma(z ; \zeta)\right) \cdot \nabla_{\xi} \Gamma(z ; \zeta)}{\Gamma^{2}(z, \zeta)} d \zeta .
$$

The properties of this operator have been studied in $[\mathbf{G L}]$. There it is proven that $u \rightarrow u_{r}$ is a smoothing operator provided that the dimension $n$ is sufficiently high. This fact, together with a device based on climbing up in the dimension allows us to prove that every $L$-superparabolic function which is the $L$-potential of a compactly supported measure, can be approximated by smooth functions of the same class, see Lemma 4.1 below.

We now wish to discuss Theorem 1.2. We begin by recalling the following beautiful result of Littman, Stampacchia and Weinberger. 
THEOREM (see $[\mathbf{L S W}])$. Let $L_{0}=\operatorname{div}(A(x) \nabla)$ be a uniformly elliptic operator with bounded measurable coefficients in $\mathbf{R}^{n}$, and let $\Omega \subset \mathbf{R}^{n}$ be a bounded open set. Then a point $x_{0} \in \partial \Omega$ is $L_{0}$-regular iff $x_{0}$ is $\Delta$-regular.

This result is based on the discovery that if $\Gamma_{0}(x, y)$ is the fundamental solution of $L_{0}$, then there exists a constant $C>0$ such that for every $x \neq y$

$$
\frac{C^{-1}}{|x-y|^{n-2}} \leq \Gamma_{0}(x, y) \leq \frac{C}{|x-y|^{n-2}} .
$$

An estimate like (1.41) is impossible for a parabolic fundamental solution. In fact, if we let $K_{\alpha_{i}}$ denote the fundamental solution of the operator $\alpha_{i} \Delta-D_{t}, \alpha_{i}>0$, $i=1,2$, then

$$
\frac{K_{\alpha_{1}}(x, t)}{K_{\alpha_{2}}(x, t)}=\left(\frac{\alpha_{2}}{\alpha_{1}}\right)^{n / 2} \exp \left(-\left(\frac{1}{\alpha_{1}}-\frac{1}{\alpha_{2}}\right) \frac{|x|^{2}}{4 t}\right), \quad x \in \mathbf{R}^{n}, t>0 .
$$

This fact has a geometrical counterpart which is enlightened by the following example due to Petrovskii (see [Pe]). For $t<0$ let $p(t)=4|t||\ln | t||$, and let $\Omega$ be the region in $\mathbf{R}^{2}$ which lies below the curve $x^{2}=4 p(t)$ and above the line $\{t=-1\}$. Then the point $(0,0) \in \partial \Omega$ is regular for $H_{2}=2 D_{x x}-D_{t}$, but it is not regular for $H=D_{x x}-D_{t}$. We remark that if $K$ and $K_{2}$ are the fundamental solutions of $\mathrm{H}$ and $\mathrm{H}_{2}$ respectively, then $K(x-y ; t-s) \leq \sqrt{2} K_{2}(x-y ; t-s)$ for every $(x, t),(y, s) \in \mathbf{R}^{2}$ with $(x, t) \neq(y, s)$. Moreover, as in [L3] one can prove

THEOREM 1.7. Let $L$ and $M$ be two operators as in Theorem 1.2. If for a given $\left(x_{0}, t_{0}\right) \in \mathbf{R}^{n+1}$

$$
A\left(x_{0}, t_{0}\right) \neq B\left(x_{0}, t_{0}\right)
$$

then there exists a bounded domain $D \subset \mathbf{R}^{n+1}$ for which $\left(x_{0}, t_{0}\right)$ is a point of $\partial D$ $L$-regular but not $M$-regular (or vice versa).

In light of the above discussion we might say that Theorem 1.2 constitutes the appropriate substitute for parabolic equations of Littman, Stampacchia and Weinberger's result.

The proof of Theorem 1.2 is based on the next theorem and on Theorem 1.3.

THEOREM 1.8. Let $L$ and $M$ be two operators as in Theorem 1.2, and let $\Gamma_{L}$ and $\Gamma_{M}$ denote their respective fundamental solutions. Let $D$ be a bounded open subset of $\mathbf{R}^{n+1}$ and suppose that for a certain $\left(x_{0}, t_{0}\right) \in \partial D$ there exist a $B>0$ and $r>0$ such that for every $(x, t)$ for which $\Gamma_{L}\left(x_{0}, t_{0} ; x, t\right) \geq(4 \pi r)^{-n / 2}$, we have

$$
\Gamma_{L}\left(x_{0}, t_{0} ; x, t\right) \leq B \Gamma_{M}\left(x_{0}, t_{0} ; x, t\right) .
$$

Then $\left(x_{0}, t_{0}\right)$ is $M$-regular if it is $L$-regular.

In proving Theorem 1.8 Wiener's criterion (Theorem 1.1 above) plays a fundamental role. We emphasize the fact that the estimate (1.44) is required to hold only in an $L$-parabolic ball with the "center" fixed at $\left(x_{0}, t_{0}\right)$.

To close this section we mention that there are in the literature several conditions for regularity which differ from that expressed in Theorem 1.1. For the heat equation Landis [La] has given a necessary and sufficient condition for regularity 
which is not, however, properly geometric. A sufficient condition similar to Landis' one has been given by Nohruzov $[\mathbf{N}]$ for operators with bounded measurable variable coefficients. Still for this kind of operators, using Wiener type series for constant coefficient operators, some sufficient conditions for regularity have been proved in [L1]. Finally, we recall the papers of Gariepy and Ziemer [GZ], and of Ziemer $[\mathbf{Z}]$, for quasi-linear operators.

2. A strong Harnack inequality. The aim of this section is to generalize to our setting a strong form of Harnack's inequality, Theorem 1.4 above, first proved by Evans and Gariepy for nonnegative solutions of the heat operator $H=\Delta-D_{t}$ in $\mathbf{R}^{n+1}$ (see [EG, Lemma 3.2]). Such a generalization is hard to come by due to the lack of an explicit knowledge of the fundamental solution, $\Gamma$, for operators with variable coefficients. However, Theorem 1.3 above serves to make up for this lack. The proof of Theorem 1.4 relies on a rather striking result, Lemma 2.1 below, whose proof occupies the biggest part of this section. In order to illustrate the idea behind it we consider the case in which the operator $L$ in (1.1) reduces to the heat operator $H$. Let then $K(x, t)=(4 \pi t)^{-n / 2} \exp \left(-|x|^{2} / 4 t\right)$ be the fundamental solution of $H$ with pole at $(0,0)$. If we set $E(x, t)=\ln K(x, t)$, we have for $t>0$

$$
\nabla_{x} E(x, t)=-\frac{x}{2 t}, \quad D_{t} E(x, t)=\frac{|x|^{2}}{4 t^{2}}-\frac{n}{2 t} \text {. }
$$

Using (2.1) it is immediate to see that given any $\Theta>1$ the inequality

$$
\left|\nabla_{x} E(x, t)\right|^{2} \leq \Theta D_{t} E(x, t)
$$

holds iff $t \leq \delta|x|^{2}$ with $\delta=\delta(\Theta)=(\Theta-1) / 2 n \Theta$, i.e., iff $(x, t)$ lies outside a paraboloid with vertex at $(0,0)$ and aperture depending on $\Theta$. This fact was first observed by Evans and Gariepy who used it in the proof of Lemma 3.2 in [EG]. Lemma 2.1 below states that if $\Gamma(x, t ; 0,0)$ is the fundamental solution of $L$ in $(1.1)$ with pole at $(0,0)$, and again we set $E(x, t)=\ln \Gamma(x, t ; 0,0)$, then $(2.2)$ has an intrinsic analogue. By this we mean that if for $\delta$ and $r$ positive we define

$$
W_{\delta, r}^{*}=\left\{\left.(x, t) \in \mathbf{R}^{n+1}\left|\Gamma(x, t ; 0,0)>(1 / 4 \pi r)^{n / 2}, t<\delta r\right| x\right|^{2}\right\},
$$

then for every $\Theta>1$ there exists a $\delta>0$ depending on $\Theta$ such that for $r$ sufficiently small

$$
\left|\nabla_{M_{t}} E(x, t)\right|_{t}^{2} \leq \Theta D_{t} E(x, t) \quad \text { for }(x, t) \in W_{\delta, r}^{*} .
$$

In (2.3) we have denoted by $\nabla_{M_{t}}$ the intrinsic gradient in the metric $g_{i j}(t) d x_{i} \otimes d x_{j}$, $g_{i j}(t)=a^{i j}(t)$ (see Theorem 1.3), and with $|\cdot|_{t}$ the intrinsic length of a vector in the same metric. (2.3), which is merely (2.13) below reformulated in intrinsic notation, plays a crucial role in the proof of the strong Harnack inequality, Theorem 1.4.

Before stating Lemma 2.1 we recall an estimate and introduce some notation. In $[\mathbf{A}]$ it was proved that there exist positive constants $\alpha_{1}, \alpha_{2}, C_{1}, C_{2}$, depending only on $\nu$ in (1.2), such that for every $(x, t),(y, s) \in \mathbf{R}^{n+1}$

$$
C_{1} K_{\alpha_{1}}(x-y ; t-s) \leq \Gamma(x, t ; y, s) \leq C_{2} K_{\alpha_{2}}(x-y ; t-s) .
$$

In (2.4) $K_{\alpha_{i}}, i=1,2$, denotes the fundamental solution of the operator $\alpha_{i} \Delta-D_{t}$. For $i=1,2$ and $C_{i}$ as in (2.4) we set for $r>0$

$$
\Omega_{r}^{i}=\Omega_{r}^{i}(0)=\left\{z \in \mathbf{R}^{n+1} \mid K_{\alpha_{i}}(-z)>C_{i}^{-1}(4 \pi r)^{-n / 2}\right\}
$$


and also $\Psi_{r}^{i}=\partial \Omega_{r}^{i}$. (2.4) implies

$$
\Omega_{r}^{1} \subset \Omega_{r} \subset \Omega_{r}^{2}
$$

$\Omega_{r}=\Omega_{r}(0)$ being the parabolic ball defined in (1.22). The heat spheres $\Psi_{r}^{i}, i=1,2$, are

$$
|x|^{2}=R_{r}^{i}(t)=2 n \alpha_{i} t \ln \left(-C_{i}^{-2 / n} \alpha_{i} r^{-1} t\right)
$$

for $-C_{i}^{2 / n} \alpha_{i}^{-1} r \leq t<0$. The functions $R_{r}^{i}(t)$ vanish at $t=0$ and at $t=$ $-C_{i}^{2 / n} \alpha_{i}^{-1} r$. We set $\eta_{i}=-C_{i}^{2 / n} \alpha_{i}^{-1}, i=1,2$. Next we choose and fix a $\sigma>0$ such that $\sigma<2 \eta_{1} \eta_{2}^{-1}$, and we let $\eta=\left(\eta_{2} \sigma+2 \eta_{1}\right) / 2$, so that $\eta_{2} \sigma<\eta<2 \eta_{1}$. We note explicitly that with this choice

$$
\inf \left\{t \mid(x, t) \in \Omega_{\sigma r}\right\} \geq-\sigma \eta_{2} r>-\eta r,
$$

since by (2.6) $\Omega_{\sigma r} \subset \Omega_{\sigma r}^{2}$ and the lowest time level of $\Omega_{\sigma r}^{2}$ is $-\sigma \eta_{2} r$. For $r>0$ we set

$$
Q_{r}=\left\{(x, t) \in \Omega_{r} \mid t>-\eta r / 2\right\} .
$$

In what follows we will use the set $Q_{2 r}$. This is the parabolic ball $\Omega_{2 r}$ with the part below the hyperplane $\{t=-\eta r\}$ removed. Because of (2.8) for every $r>0$ we have $\Omega_{\sigma r} \subset Q_{2 r}$.

We remark that the functions $R_{r}^{1}, i=1,2$, in (2.6) attain their maximum at $t_{i}=-C_{1}^{2 / n} \alpha_{i}^{-1} e^{-1} r, i=1,2$, where $R_{r, \max }^{i}=R_{r}^{i}\left(t_{i}\right)=2 n e^{-1} C_{i}^{2 / n} r, i=1,2$. Therefore, the set $\Omega_{r}$ is contained in the parabolic cylinder

$$
C_{r}=\left\{\left.(x, t) \in \mathbf{R}^{n+1}|| x\right|^{2} \leq 2 n e^{-1} C_{2}^{2 / n} r,-C_{2}^{2 / n} \alpha_{2}^{-1} r \leq t \leq 0\right\} .
$$

For $\delta$ and $r>0$ we define

$$
P_{\delta, r}=\left\{\left.(x, t) \in \mathbf{R}^{n+1}|t \leq-\delta r| x\right|^{2}\right\} .
$$

The set $P_{\delta, r}$ is the interior of the paraboloid $\left\{t=-\delta r|x|^{2}\right\}$ with vertex at $(0,0)$ and opening in the negative time direction. Finally, we introduce the open set

$$
W_{\delta, r}=\Omega_{\sigma r} \backslash P_{\delta, r} .
$$

In the proof of Lemma 2.1 below we will find it convenient to work with the operator (1.1), rather than its adjoint $L^{*}$. For this reason we define for any of the sets $\Omega_{\sigma r}$, $Q_{r}, C_{r}, P_{\delta, r}, W_{\delta, r}$ introduced above a corresponding starred one, $\Omega_{\sigma r}^{*}, Q_{r}^{*}, C_{r}^{*}, P_{\delta, r}^{*}$, $W_{\delta, r}^{*}$. The starred set is the image of the original one under the time-reflection $(x, t) \rightarrow(x,-t)$. Thus, e.g., $P_{\delta, r}^{*}=\left\{\left.(x, t) \in \mathbf{R}^{n+1}|t \geq \delta r| x\right|^{2}\right\}$.

LEMMA 2.1. There exist $r_{0}>0$, and for every $\Theta>1$ a $\delta=\delta(\Theta)>0$ such that if $\Gamma=\Gamma(z ; 0)$ is the fundamental solution of $L$ in $(1.1)$ with pole at $(0,0)$, then

$$
\frac{A \nabla_{x} \Gamma \cdot \nabla_{x} \Gamma}{\Gamma^{2}} \leq \Theta \frac{D_{t} \Gamma}{\Gamma} \quad \text { in } W_{\delta, r}^{*}
$$

for all $r \leq r_{0}$.

PROOF. It is based on Theorem 1.3. We begin be remarking that (2.13) can be rewritten in the intrinsic notation of Theorem 1.3 as

$$
\left|\nabla_{M_{t}} \Gamma\right|_{t}^{2}=\left\langle\nabla_{M_{t}} \Gamma, \nabla_{M_{t}} \Gamma\right\rangle_{t} \leq \Theta \Gamma \cdot D_{t} \Gamma \quad \text { in } W_{\delta, r}^{*}
$$


For $t \geq 0$ fixed we have denoted by $M_{t}$ the manifold $\left(K_{r_{0}}, g_{i j}(t)\right)$, where $K_{r_{0}}=$ $\left\{\left.x \in \mathbf{R}^{n}|| x\right|^{2}<2 n e^{-1} C_{2}^{2 / n} r_{0}\right\}$ (see (2.10)), and $r_{0}$ will be fixed, conveniently small, later. At this point we fix a $k \in \mathrm{N}$ large enough in Theorem $1.3(k>n / 2+10$ will do!) so that we can write for $z \in C_{r_{0}}$ (see (2.10)),

$$
\left\{\begin{array}{l}
\Gamma(z ; 0)=\gamma_{k}(z)+w_{k}(z) \\
D_{j} \Gamma(z ; 0)=D_{j} \gamma_{k}(z)+w_{k, j}(z), \quad j=1, \ldots, n \\
D_{t} \Gamma(z ; 0)=D_{t} \gamma_{k}(z)+\bar{w}_{k}(z)
\end{array}\right.
$$

In $(2.15)$ we have set $\gamma_{k}(z)=G(z ; 0) \sum_{j=0}^{k} t^{j} u_{j}(z)$, where $G(z ; 0)=G(x, 0, t)=$ $(4 \pi t)^{-n / 2} \exp \left(-d^{2}(x, 0, t) / 4 t\right)$ is the generalized Gaussian and the $u_{j}$ 's are the functions appearing in (1.18). Moreover, $w_{k}, w_{k, j}, j=1, \ldots, n$, and $\bar{w}_{k}$ are $(n+2)$ functions in $C_{r}$ such that

$$
w_{k}(z)=o\left(r_{0}\right), \quad w_{k, j}(z)=o\left(r_{0}\right), \quad w_{k}(z)=o\left(r_{0}\right)
$$

as $r_{0} \rightarrow 0$.

If we set $\vec{w}_{k}=\left(w_{k, 1}, \ldots, w_{k, n}\right)$ we can rewrite the second equation in (2.15) as

$$
\nabla_{M_{t}} \Gamma(z ; 0)=\nabla_{M_{t}} \gamma_{k}(z)+A(t) \vec{w}_{k}(z)
$$

$A(t)$ being the matrix $\left(a_{i j}(t)\right)=\left(g_{i j}(t)\right)^{-1}$. Henceforth, we will use the notation $|\cdot|_{t}^{2}=\langle\cdot, \cdot\rangle_{t}$ for the square of the Riemannian length in $M_{t}$. We will also take the freedom of switching, when needed, from Euclidean to intrinsic notation, and vice versa. Finally, we set

$$
\eta_{k}(z)=\sum_{j=0}^{k} t^{j} u_{j}(z)
$$

We can then write

$$
\eta_{k}^{2}(z)=\left(u_{0}+t \sum_{j=0}^{k} t^{j-1} u_{j}\right)^{2}=u_{0}^{2}+t \chi_{k}
$$

where $\chi_{k} \in C^{\infty}\left(C_{r_{0}}\right)$. We are now ready to prove (2.14).

Using (2.17), recalling that $\gamma_{k}=G \eta_{k}$, we obtain for the left-hand side of (2.14)

$$
\left\langle\nabla_{M_{t}} \Gamma, \nabla_{M_{t}} \Gamma\right\rangle_{t}=\left|\nabla_{M_{t}} \Gamma\right|_{t}^{2}=\left|\nabla_{M_{t}} \gamma_{k}\right|_{t}^{2}+2\left\langle\nabla_{M_{t}} \gamma_{k}, A(t) \vec{w}_{k}\right\rangle_{t}+\left|A(t) \vec{w}_{k}\right|_{t}^{2}
$$

Now the fact that

$$
\nabla_{M_{t}} G=-\frac{1}{4 t} G \nabla_{M_{t}} d^{2}, \quad \nabla G=-\frac{1}{4 t} G \nabla d^{2}
$$


and a computation give

$$
\begin{aligned}
\left|\nabla_{M_{t}} \Gamma\right|_{t}^{2}= & \frac{d^{2}}{4 t^{2}} G^{2} \eta_{k}^{2}-\frac{d}{t} G^{2} \eta_{k}\left\langle\nabla_{M_{t}} d, \nabla_{M_{t}} \eta_{k}\right\rangle_{t} \\
& +G^{2} A(t) \nabla \eta_{k} \cdot \nabla \eta_{k}+2 G A(t) \nabla \eta_{k} \cdot \vec{w}_{k} \\
& \quad-\frac{d}{t} G \eta_{k}\left\langle\nabla_{M_{t}} d, A(t) \vec{w}_{k}\right\rangle_{t}+A(t) \vec{w}_{k} \cdot \vec{w}_{k} \\
= & \frac{d^{2}}{4 t^{2}} G^{2}\left\{u_{0}^{2}+t\left[\chi_{k}+\frac{4 t}{d^{2}} A(t) \nabla \eta_{k}\right.\right. \\
& \left.\quad+\frac{8 t}{d^{2}} \frac{1}{G} A(t) \nabla \eta_{k} \cdot \vec{w}_{k}+\frac{4 t}{d^{2} G^{2}} A(t) \vec{w}_{k} \cdot \vec{w}_{k}\right] \\
& \left.\quad+d\left[\frac{4 t}{d^{2}} \eta_{k} D_{d}\left(\eta_{k}\right)-\frac{4 t}{d^{2}} \frac{1}{G} \eta_{k} A(t) \nabla d \cdot \vec{w}_{k}\right]\right\} .
\end{aligned}
$$

In passing from the first to the second equality in (2.22) we have used the fact that if $f=f(d)$ depends only on the radial coordinate $d$, and $g$ is a $C^{1}$ function on $M_{t}$, then $\left\langle\nabla_{M_{t}} f, \nabla_{M_{t}} g\right\rangle_{t}=f^{\prime}(d) D_{d} g$, where $D_{d}=\partial / \partial d$. We recall now that on $\Omega_{r_{0}} \Gamma>\left(4 \pi r_{0}\right)^{-n / 2}$, hence $\Gamma^{-1}<\left(4 \pi r_{0}\right)^{n / 2}$. By the first equation in $(2.15)$ $\Gamma=G \eta_{k}+w_{k}$, where $\eta_{k}(z) \rightarrow u_{0}=1$ and $w_{k}(z) \rightarrow 0$ as $z \in C_{r_{0}}$ and $r_{0} \rightarrow 0$. Therefore, $G^{-1}(z)=O\left(r_{0}^{n / 2}\right)$ as $z \in \Omega_{r_{0}}$ and $r_{0} \rightarrow 0$. These considerations allow us to write $(2.22)$ as

$$
\left|\nabla_{M_{t}} \Gamma\right|_{t}^{2}=\frac{d^{2}}{4 t^{2}} G^{2}\left\{u_{0}^{2}+t h+d k\right\}
$$

where $h(z)=O(1)$ and $k(z)=O(1)$, as $z \in W_{\delta, r}$ and $r_{0} \rightarrow 0$.

We now look at the right-hand side of (2.14). The first and third equation in (2.15) and the identity

$$
D_{t} G=\left(d^{2} / 4 t^{2}-n / 2 t-D_{t} d^{2} / 4 t\right) G
$$

give

$$
\begin{aligned}
\Gamma \cdot D_{t} \Gamma=\gamma_{k} D_{t} \gamma_{k}+\gamma_{k} \bar{w}_{k}+D_{t} \gamma_{k} \bar{w}_{k}+w_{k} \bar{w}_{k} \\
=G^{2}\left(\eta_{k}+\frac{w_{k}}{G}\right)\left[\left(\frac{d^{2}}{4 t^{2}}-\frac{n}{2 t}-\frac{1}{2 t} D_{t} d^{2}\right) \eta_{k}+D_{t} \eta_{k}+\frac{\bar{w}_{k}}{G}\right] \\
=\frac{d^{2}}{4 t^{2}} G^{2}\left\{u_{0}^{2}\left(1+\frac{w_{k}}{\eta_{k} G}\right)-\frac{t}{d^{2}}\left(\eta_{k}^{2}+\frac{w_{k}}{G} \eta_{k}\right)\left(2 n+D_{t} d^{2}\right)\right. \\
+t\left[\chi_{k}\left(1+\frac{w_{k}}{\eta_{k} G}\right)\right. \\
\left.\left.\quad+\frac{4 t}{d^{2}}\left(\eta_{k} D_{t} \eta_{k}+\frac{\eta_{k} \bar{w}_{k}}{G}+\frac{w_{k} D_{t} \eta_{k}}{G}+\frac{w_{k} \bar{w}_{k}}{G^{2}}\right)\right]\right\} .
\end{aligned}
$$

Considerations similar to those made before (2.23) lead us to rewrite (2.25) as

$$
\Gamma \cdot D_{t} \Gamma=\frac{d^{2}}{4 t^{2}} G^{2}\left\{u_{0}^{2} \varphi-\frac{t}{d^{2}} \Psi+t \chi\right\},
$$


where $\varphi(z) \rightarrow 1, \Psi(z)=O(1)$ and $\chi(z)=O(1)$, as $z \in \Omega_{r}$ and $r_{0} \rightarrow 0$. At this point, using (2.23) and (2.26) we can prove (2.14): it will be sufficient to show that there exist $r_{0}$ small enough and a $\delta=\delta(\Theta)>0$ such that for every $r \in\left(0, r_{0}\right]$

$$
u_{0}^{2}+t h+d k \leq \Theta\left(u_{0}^{2} \varphi-\frac{t}{d^{2}} \Psi+t \chi\right) \quad \text { in } W_{\delta, r}^{*} .
$$

(2.27) is equivalent to

$$
\Theta \frac{t}{d^{2}} \Psi \leq(\Theta \varphi-1) u_{0}^{2}+t(\Theta \chi-h)-d k \text { in } W_{\delta, r}^{*} .
$$

Since $(\Theta \varphi(z)-1) u_{0}^{2}(t) \rightarrow \Theta-1$ as $z \in \Omega_{r}$ and $r_{0} \rightarrow 0$, it is clear that we can find $r_{0}>0$ sufficiently small such that

$$
(\Theta \varphi-1) u_{0}^{2}+t(\Theta \chi-h)-d k \geq(\Theta-1) / 2 \text { on } \Omega_{r}
$$

for every $r \in\left(0, r_{0}\right]$. Now let $M=\sup _{\Omega_{r_{0}}}|\psi|$. Using the fact that there exist two positive constants $\alpha, \beta$ such that for every $x, y, t$

$$
\alpha|x-y|^{2} \leq d^{2}(x, y, t) \leq \beta|x-y|^{2},
$$

it is clear that (2.28) will hold if we choose $\delta>0$ in the definition (2.11) of $P_{\delta, r}$ such that $\delta=\alpha(\Theta-1) / 2 \Theta M$, with $\alpha$ as in (2.30). This completes the proof of Lemma 2.1 .

We are now ready to give the

ProOF OF THEOREM 1.4. We recall (see (1.26)) that $Q_{2 r}$ is the set $\{(x, t) \in$ $\left.\Omega_{2 r} \mid t>-\eta r\right\}$ (see the beginning of this section). Let then $u>0$ be as in the statement of Theorem 1.4. By homogeneity we may (and do) assume that

$$
f_{I_{r}} u(x,-\eta r) d x=1
$$

where $I_{r}$ is defined in (1.27). To prove (1.28) we must then show that there exists a $\Lambda>0$ such that

$$
\inf _{\Omega_{(3 / 4) \text { or }}} u>\Lambda^{-1} .
$$

We now fix $r_{0}>0$ and a $\delta>0$ such that (2.13) in Lemma 2.1 holds corresponding to the choice $\gamma=4 / 3$. Estimating $u$ from below in that part of $\Omega_{(3 / 4) \sigma r}$ which lies in $P_{\delta, r}$ (see (2.11)) is an easy matter. Because of (2.32) and the time-lag (2.8), Harnack's inequality assures the existence of a constant $\varepsilon>0$, independent of $r$, such that for $r \leq r_{0}$

$$
\inf \left\{u(z) \mid z \in \Omega_{\sigma r} \cap P_{\delta, r}\right\} \geq \varepsilon .
$$

The difficult part of the proof is to estimate $u$ in that part of $\Omega_{(3 / 4) \sigma r}$ which lies outside $P_{\delta, r}$, i.e., in the set $W_{\delta, r}$ defined in (2.12). But we have already done the hard work in proving Lemma 2.1. At this point we follow an idea in [EG]: we construct a positive function $v$ that vanishes on the parabolic sphere $\Psi_{\sigma r}$ and is bounded from above along $\Omega_{\sigma r} \cap \partial P_{\delta, r}$. Lemma 2.1 will then prove that the constructed $v$ is a subsolution. As in [EG] we take

$$
\Psi(s)=\arctan (s+16)-\arctan (16),
$$


so that for $s \in \mathbf{R}^{+}$

$$
\begin{cases}\Psi(0)=0, & 0 \leq \Psi(s)<\pi / 2 \\ \Psi^{\prime}(s)>0, & 0 \leq-\Psi^{\prime \prime}(s) \leq \frac{1}{8} \Psi^{\prime}(s) .\end{cases}
$$

Now we set

$$
v(z)=\Psi\left(\ln \left[(4 \pi \sigma r)^{n / 2} \Gamma(0 ; z)\right]\right),
$$

where $\Gamma(0 ; z)$ is the fundamental solution of $L^{*}=\operatorname{div}\left(A(z) \nabla_{x}\right)+D_{t}$ with pole at $0=(0,0) \in \mathbf{R}^{n+1}$. By $(1.21)$ it is obvious that

$$
v(z) \equiv 0 \quad \text { for every } z \in \Psi_{\sigma r} \backslash\{(0,0)\} .
$$

Also by (2.35)

$$
v(z) \leq \pi / 2 \quad \text { for } z \in \bar{\Omega}_{\sigma r} \cap \partial P_{\delta, r} .
$$

At this point we

Claim. $L v(z) \geq 0$ for $z \in W_{\delta, r}$.

Let us assume for a moment the claim is true. Then by (2.33), (2.37), (2.38), the fact that $u \geq 0$ in $\Psi_{\sigma r} \backslash\{(0,0)\}$, and the maximum principle we would infer that

$$
u \geq c v \quad \text { in } W_{\delta, r}
$$

with $c=2 \varepsilon / \pi, \varepsilon$ as in $(2.33)$. Since $\Gamma(0 ; z)>(3 \pi \sigma r)^{-n / 2}$ on $\Omega_{(3 / 4) \sigma r} \backslash P_{\delta, r},(2.39)$ would finally give

$$
\inf _{\Omega_{(3 / 4) \sigma r} \backslash P_{\delta, r}} u \geq c \inf _{\Omega_{(3 / 4) \sigma r} \backslash P_{\delta, r}} v \geq C \Psi\left(\ln \left(\frac{4}{3}\right)^{n / 2}\right),
$$

which, together with (2.33), implies (2.32). Therefore, to complete the proof of the theorem we only have to prove the claim. To this end we observe that proving the claim is equivalent to proving that if we set

$$
w(z)=\Psi\left(\ln \left[(4 \pi \sigma r)^{n / 2} \Gamma(z ; 0)\right]\right),
$$

then

$$
L^{*} w \geq 0 \quad \text { in } W_{\delta, r}^{*}
$$

Now a computation yields

$$
\begin{aligned}
L^{*} w & =\frac{\Psi^{\prime}}{\Gamma} L \Gamma+\left(4 \Psi^{\prime \prime}-\Psi^{\prime}\right) \frac{A \nabla_{x} \Gamma \cdot \nabla_{x} \Gamma}{\Gamma^{2}}+2 \Psi^{\prime} \frac{D_{t} \Gamma}{\Gamma} \\
& =\left(4 \Psi^{\prime \prime}-\Psi^{\prime}\right) \frac{A \nabla_{x} \Gamma \cdot \nabla_{x} \Gamma}{\Gamma^{2}}+2 \Psi^{\prime} \frac{D_{t} \Gamma}{\Gamma}
\end{aligned}
$$

where in the last equality we have used the fact that $L(\Gamma(z ; 0))=0$ for $z \neq(0,0)$. Because of $(2.35) \Psi^{\prime}-4 \Psi^{\prime \prime} \leq 3 \Psi^{\prime} / 2$. Therefore (2.42) will be true if

$$
\frac{A \nabla_{x} \Gamma \cdot \nabla_{x} \Gamma}{\Gamma^{2}} \leq \frac{4}{3} \frac{D_{t} \Gamma}{\Gamma} \text { in } W_{\delta, r}^{*}
$$

for all $r$ sufficiently small. But this is (2.13) with $\Theta=4 / 3$, thus the claim follows from Lemma 2.1. 
3. Mean value formulas for solutions of $L u=f$ and their consequences. In this section we will generalize to solutions of the inhomogeneous equation $L u=f$ some weighted average formulas for solutions of $L u=0$ that were found in [FG] (see Theorem 1.5 in $\S 1$ ). Then we will study the derivatives of these averages as functions of the radius (see Theorem 1.6 and Corollary 1.2). (1.38) above extends to our setting well-known mean value and sub- (super-)mean value properties of harmonic and sub- (super-)harmonic functions. It is an immediate corollary of it that if $L u \geq 0$, then its "average" (1.36) is an increasing function of the radius. Corollary 1.1 generalizes to the case of operators with variable coefficients part (c) of Lemma 3.1 in [EG]. It will play an important role in the proof of the sufficiency of Wiener's criterion in $\S 4$.

In what follows $L$ will denote the parabolic operator introduced in (1.1). We will indicate the points $(x, t),\left(x_{0}, t_{0}\right),(\xi, \tau)$ in $\mathbf{R}^{n+1}$ respectively with the letters $z, z_{0}$, S. $\Gamma\left(z_{0}, z\right)=\Gamma\left(x_{0}, t_{0} ; x, t\right)$ stands for the fundamental solution of $L$. As a function of $z, \Gamma\left(z_{0} ; z\right)$ is the fundamental solution of the backward operator

$$
L^{*}=\operatorname{div}\left(A(z) \nabla_{x}\right)+D_{t}
$$

with pole at $z=z_{0}$. Sard's theorem and the fact that $\Gamma\left(z_{0} ; \cdot\right) \in C^{\infty}\left(\mathbf{R}^{n+1} \backslash\left\{z_{0}\right\}\right)$ imply that for a.e. $r>0$ the set $\Psi_{r}\left(z_{0}\right) \backslash\left\{z_{0}\right\}$ defined by (1.21) is a smooth regular $n$ dimensional manifold in $\mathbf{R}^{n+1}$. Moreover if $\Omega_{r}\left(z_{0}\right)$ is as in (1.22) $\partial \Omega_{r}\left(z_{0}\right)=\Psi_{r}\left(z_{0}\right)$. It was proven in [FG] that: if $u$ is a (smooth) solution to $L u=0$ and if $z_{0} \in \mathbf{R}^{n+1}$, then

(i) for a.e. $r>0$

$$
u\left(z_{0}\right)=\int_{\Psi_{r}\left(z_{0}\right)} u(z) \frac{A(z)\left(\nabla_{x} \Gamma\left(z_{0} ; z\right)\right) \cdot \nabla_{x} \Gamma\left(z_{0} ; z\right)}{\left|\left(\nabla_{x} \Gamma\left(z_{0} ; z\right), D_{t} \Gamma\left(z_{0} ; z\right)\right)\right|} d H_{n}(z) ;
$$

(ii) for every $r>0$

$$
u\left(z_{0}\right)=(4 \pi r)^{-n / 2} \int_{\Omega_{r}\left(z_{0}\right)} u(z) \frac{A(z)\left(\nabla_{x} \Gamma\left(z_{0} ; z\right)\right) \cdot \nabla_{x} \Gamma\left(z_{0} ; z\right)}{\Gamma^{2}\left(z_{0} ; z\right)} d z .
$$

In (3.2) and (3.3), denotes the inner product in $\mathbf{R}^{n}$, while in (3.3) $\left|\left(\nabla_{x} \Gamma, D_{t} \Gamma\right)\right|$ denotes the Euclidean length in $\mathbf{R}^{n+1}$ of the $(n+1)$-dimensional vector $\left(\nabla_{x} \Gamma, D_{t} \Gamma\right)$, and $d H_{n}$ the $n$-dimensional Hausdorff measure. Theorem 1.5 extends (3.2) and (3.3) to solutions of the inhomogeneous equation $L u=f$. Its proof is modelled on the arguments in [FG] so that we will try to avoid repetitions as much as we can without affecting readability. An important tool in [FG] was the so-called co-area formula of Federer (see [Fe, Theorem 3.2.12, p. 249]). This states that: if $f \in L^{1}\left(\mathbf{R}^{n+1}\right)$ and $g \in \operatorname{Lip}\left(\mathbf{R}^{n+1}\right)$, then

$$
\int_{\mathbf{R}^{n+1}} f(z)\left|\left(\nabla_{x} g, D_{t} g\right)\right| d z=\int_{-\infty}^{\infty} d \alpha\left(\int_{g=\alpha} f(z) d H_{n}(z)\right) .
$$

PROOF OF THEOREM 1.5. The proof of (1.30) imitates that given in [FG] of the above-mentioned surface formula (3.2). We therefore omit the details and refer the reader to that source. As for the proof of (1.31) we will only indicate how Federer's formula (3.4) can be used to obtain (1.31) from (1.30). Setting $l=r$ in 
(1.30), multiplying both sides by $l^{n / 2-1}$ and integrating between 0 and $r$ gives

$$
\begin{aligned}
& -\int_{0}^{r} l^{n / 2} \int_{\Gamma=(4 \pi l)^{-n / 2}} u(\varsigma) A(\varsigma)\left(\nabla_{\xi} \Gamma\left(z_{0} ; \varsigma\right)\right) \cdot \vec{N}_{\xi}(\varsigma) d H_{n}(\varsigma) \frac{d l}{l} \\
& \quad=\frac{2}{n} u\left(z_{0}\right) r^{n / 2}+\int_{0}^{r} l^{n / 2} \int_{\Gamma>(4 \pi l)^{-n / 2}} L u(z)\left[\Gamma\left(z_{0} ; z\right)-(4 \pi r)^{-n / 2}\right] d z \frac{d l}{l}
\end{aligned}
$$

In (3.5) with abuse of notation we have denoted by $\Gamma=(4 \pi l)^{-n / 2}$ and $\Gamma>$ $(4 \pi l)^{-n / 2}$, respectively, the sets $\Psi_{l}\left(z_{0}\right)$ and $\Omega_{l}\left(z_{0}\right)$. Now observe that $(3.4)$ can be rewritten

$$
\int_{\mathbf{R}^{n+1}} f(z) d z=\int_{-\infty}^{\infty} d \alpha\left(\int_{g=\alpha} \frac{f(z)}{\left(\nabla_{x} g, D_{t} g\right) \mid} d H_{n}(z)\right) .
$$

Using (3.6) in the left-hand side of (3.5), recalling that $\vec{N}_{\xi}=-\nabla_{\xi} \Gamma /\left|\left(\nabla_{\xi} \Gamma, D_{t} \Gamma\right)\right|$, yields

$$
\begin{aligned}
& -\int_{0}^{r} l^{n / 2} \int_{\Gamma=(4 \pi l)^{-n / 2}} u(\varsigma) A(\varsigma)\left(\nabla_{\xi} \Gamma\left(z_{0} ; \varsigma\right)\right) \cdot \vec{N}_{\xi}(\varsigma) d H_{n}(\varsigma) \frac{d l}{l} \\
& =\frac{2}{n}\left(\frac{1}{4 \pi}\right)^{n / 2} \int_{(4 \pi r)^{-n / 2}}^{\infty} \alpha^{-2} \int_{\Gamma=\alpha} u(\varsigma) \frac{A(\varsigma)\left(\nabla_{\xi} \Gamma\left(z_{0} ; \zeta\right)\right) \cdot \nabla_{\xi} \Gamma\left(z_{0} ; \zeta\right)}{\left|\left(\nabla_{\xi} \Gamma\left(z_{0} ; \zeta\right), D_{t} \Gamma\left(z_{0} ; \zeta\right)\right)\right|} d H_{n}(\varsigma) d \alpha \\
& =\frac{2}{n}\left(\frac{1}{4 \pi}\right)^{n / 2} \int_{\Gamma>(4 \pi r)^{-n / 2}} u(z) \frac{A(z)\left(\nabla_{x} \Gamma\left(z_{0} ; z\right)\right) \cdot \nabla_{x} \Gamma\left(z_{0} ; z\right)}{\Gamma^{2}\left(z_{0} ; z\right)} d z
\end{aligned}
$$

Inserting (3.7) in (3.5) gives (1.31).

We now introduce the following weighted averages of a function $u \in C^{\infty}\left(\mathbf{R}^{n+1}\right)$ :

$$
u_{r}\left(z_{0}\right)=(4 \pi r)^{-n / 2} \int_{\Omega_{r}\left(z_{0}\right)} u(z) \frac{A(z)\left(\nabla_{x} \Gamma\left(z_{0} ; z\right)\right) \cdot \nabla_{x} \Gamma\left(z_{0} ; z\right)}{\Gamma^{2}\left(z_{0} ; z\right)} d z
$$

where $z_{0} \in \mathbf{R}^{n+1}$ and $r>0$. Using (3.8) we might rewrite (1.31) as

$$
u_{r}\left(z_{0}\right)=u\left(z_{0}\right)+\frac{n}{2} r^{-n / 2} \int_{0}^{r} l^{n / 2} \int_{\Omega_{l}\left(z_{0}\right)} L u(z)\left[\Gamma\left(z_{0} ; z\right)-(4 \pi l)^{-n / 2}\right] d z \frac{d l}{l}
$$

We are now ready to give the

PROOF OF THEOREM 1.6. Differentiating (3.9) gives

$$
\begin{aligned}
\frac{d}{d r} u_{r}\left(z_{0}\right)= & -\left(\frac{n}{2}\right)^{2} r^{-n / 2-1} \int_{0}^{r} l^{n / 2} \int_{\Omega_{l}\left(z_{0}\right)} L u(z)\left[\Gamma\left(z_{0} ; z\right)-(4 \pi l)^{-n / 2}\right] d z \frac{d l}{l} \\
& +\frac{n}{2} r^{-1} \int_{\Omega_{r}\left(z_{0}\right)} L u(z)\left[\Gamma\left(z_{0} ; z\right)-(4 \pi r)^{-n / 2}\right] d z .
\end{aligned}
$$


Now, by Tonelli's theorem

$$
\begin{aligned}
\int_{0}^{r} l^{n / 2} & \int_{\Omega_{l}\left(z_{0}\right)} L u(z)\left[\Gamma\left(z_{0} ; z\right)-(4 \pi l)^{-n / 2}\right] d z \frac{d l}{l} \\
= & \int_{\Omega_{r}\left(z_{0}\right)} L u(z) \int_{\left((4 \pi)^{n / 2} \Gamma\left(z_{0} ; z\right)\right)^{-2 / n}}^{r} l^{n / 2}\left[\Gamma\left(z_{0} ; z\right)-(4 \pi l)^{-n / 2}\right] \frac{d l}{l} d z \\
= & \int_{\Omega_{r}\left(z_{0}\right)} L u(z)\left[\Gamma\left(z_{0} ; z\right) 2 n^{-1} l^{n / 2}-(4 \pi)^{-n / 2} \ln l\right]_{l=\left((4 \pi)^{n / 2} \Gamma\right)^{-2 / n}}^{l=r} d z \\
= & \frac{2}{n} r^{n / 2} \int_{\Omega_{r}\left(z_{0}\right)} L u(z) \Gamma\left(z_{0} ; z\right) d z-\frac{2}{n}(4 \pi)^{-n / 2} \int_{\Omega_{r}\left(z_{0}\right)} L u(z) d z \\
& -\frac{2}{n}(4 \pi)^{-n / 2} \int_{\Omega_{r}\left(z_{0}\right)} L u(z) \ln \left[(4 \pi r)^{n / 2} \Gamma\left(z_{0} ; z\right)\right] d z .
\end{aligned}
$$

Inserting (3.11) in (3.10) and simplifying finally gives (1.37).

REMARK 3.1. The remarkable feature of equation (1.37) is that the kernel $\ln \left[(4 \pi r)^{n / 2} \Gamma\left(z_{0} ; z\right)\right]$ appearing in it is strictly positive. This is so since $\Gamma\left(z_{0} ; z\right)>$ $(4 \pi r)^{-n / 2}$ on $\Omega_{r}\left(z_{0}\right)$. As a consequence, the sign of the derivative $(d / d r) u_{r}\left(z_{0}\right)$ depends solely on the sign of $L u$. Hence, if $L u \geq 0$, i.e., if $u$ is a subsolution of $L$, then $(d / d r) u_{r}\left(z_{0}\right) \geq 0$, a result which extends to our setting well-known properties of subharmonic functions.

PROOF OF COROLLARY 1.2. We have by (1.37)

$$
\begin{aligned}
& u_{\alpha^{2} r}\left(z_{0}\right)-u_{\alpha r}\left(z_{0}\right)=\int_{\alpha r}^{\alpha^{2} r} \frac{d}{d s} u_{s}\left(z_{0}\right) d s \\
& \quad=\frac{n}{2}(4 \pi)^{-n / 2} \int_{\alpha r}^{\alpha^{2} r} s^{-n / 2} \int_{\Omega_{s}\left(z_{0}\right)} L u(z) \ln \left[(4 \pi s)^{n / 2} \Gamma\left(z_{0} ; z\right)\right] d z \frac{d s}{s} \\
& \quad \geq \frac{n}{2}(4 \pi)^{-n / 2} \int_{\Omega_{r}\left(z_{0}\right)} L u(z) \ln \left[(4 \pi x r)^{n / 2} \Gamma\left(z_{0} ; z\right)\right] d z\left(\int_{\alpha r}^{\alpha^{2} r} s^{-n / 2} \frac{d s}{s}\right) .
\end{aligned}
$$

Now $(4 \pi r)^{n / 2} \Gamma\left(z_{0} ; z\right)>1$ on $\Omega_{r}\left(z_{0}\right)$, hence (3.12) yields (1.38).

We wish to close this section observing explicitly that if $A(z) \equiv$ Identity, and therefore $L=H=\Delta-D_{t}$, the heat operator in $\mathbf{R}^{n+1}$, then

$$
\Gamma\left(z_{0} ; z\right)=\left[4 \pi\left(t_{0}-t\right)\right]^{-n / 2} \exp \left(-\left|x_{0}-x\right|^{2} / 4\left(t_{0}-t\right)\right),
$$

the Gauss-Weierstrass kernel in $\mathbf{R}^{n+1}$. In this case if $z_{0}=(0,0)$

$$
\ln \left[(4 \pi r)^{n / 2} \Gamma(0, z)\right]=\frac{|x|^{2}-2 n t \ln (-t / r)}{4 t},
$$

so that (1.37) becomes

$$
\frac{d}{d r} u_{r}\left(z_{0}\right)=\frac{n}{2 r}(4 \pi r)^{-n / 2} \int_{\Omega_{r}(0)} H u(z)\left[\frac{|x|^{2}-2 n t \ln (-t / r)}{4 t}\right] d z .
$$

(3.14) is, up to constants, the formula found by Evans and Gariepy (see part (b) of Lemma 3.1 in [EG]) by a direct computation, possible for them because of the explicit knowledge of $\Gamma$. 
4. Wiener's criterion and its consequences. The main goal of this section is to provide the proof of Theorems 1.1, 1.2 and 1.9. Before starting, however, we need to recall some facts from potential theory. For a compact set $F \subset \mathbf{R}^{n+1}$ we define for $z \in \mathbf{R}^{n+1}$

$$
\begin{gathered}
v_{F}^{L}(z)=\inf \{u(z) \mid u \text { is } L \text {-superparabolic, } u \geq 1 \text { on } F, u \geq 0\}, \\
V_{F}^{L}(z)=\frac{\lim }{\varsigma \rightarrow z} v_{F}^{L}(\varsigma) .
\end{gathered}
$$

$V_{F}^{L}$ is called the $L$-equilibrium potential of $F$. In what follows, when no confusion will arise, we will simply denote $v_{F}^{L}$ and $V_{F}^{L}$ by $v_{F}$ and $V_{F}$, respectively. In a standard fashion (see, e.g., [L1]), it can be proved that there exists a unique $\mu \in$ $M^{+}(\partial F)$, called the L-equilibrium measure of $F$, such that

$$
\Gamma_{\mu}(z)=V_{F}(z), \quad z \in \mathbf{R}^{n+1},
$$

and

$$
\mu\left(\mathbf{R}^{n+1}\right)=\operatorname{cap}_{L}(F)
$$

Moreover

$$
0 \leq V_{F} \leq 1, \quad \text { and } \quad V_{F}(z) \equiv 1 \text { for } z \in \stackrel{\circ}{F} \text {. }
$$

In the course of the proof of Theorem 1.1 below we will work only with bounded $L$-superparabolic functions which are potentials $\Gamma_{\mu}$ of some $\mu \in M^{+}(F)$, with $F$ a compact subset of $\mathbf{R}^{n+1}$. From now on we will denote by $P$ the class of all such functions, i.e.,

$$
\begin{aligned}
& P=\left\{u L \text {-superparabolic } \mid u \in L^{\infty}\left(\mathbf{R}^{n+1}\right), u=\Gamma_{\mu}\right. \\
& \text { for some } \left.\mu \in M^{+}(F), F \subset \mathbf{R}^{n+1} \text { compact }\right\} .
\end{aligned}
$$

The class $P$ enjoys a remarkable approximation property, which is expressed by the following

LEMMA 4.1. Let $u=\Gamma_{\mu} \in P$. Then there exists a sequence $\left(u_{j}\right)_{j \in \mathbf{N}}$, with $u_{j} \in P \cap C^{2}\left(\mathbf{R}^{n+1}\right)$ and $u_{j} \leq u_{j+1}$ for every $j \in \mathbf{N}$, such that $\lim _{j \rightarrow \infty} u_{j}=u$. Moreover, $\left(u_{j}\right)_{j \in \mathbf{N}}$ can be chosen such that

$$
\operatorname{supp} \mu=\bigcap_{j \in \mathbf{N}} \operatorname{supp}\left(L u_{j}\right)
$$

and that for every $\delta>0$ there exists $p \in \mathbf{N}$ for which $u_{j}(z) \equiv u(z)$ for every $j \geq p$ and any $z$ such that $\operatorname{dist}(z, \operatorname{supp} \mu) \geq \delta$.

We do not present here the rather long proof of Lemma 4.1, but refer to [GL] for it. We only mention that the possibility of approximating any potential with a smooth one ultimately relies on the regularizing properties of the parabolic averaging operator introduced in (1.40).

Hereafter, we will adopt the notation of $\S 2$. We fix once and forever a bounded open subset $D \subset \mathbf{R}^{n+1}$ as in Theorems $1.1,1.2$, and 1.8, and we will assume from now on that the point $z_{0}=\left(x_{0}, t_{0}\right) \in \partial D$ in those theorems coincides with the origin. This is not restrictive. 
Proposition 4.1. With $C_{r}$ defined by $(2.10), z_{0}$ is $L$-regular for $D$ iff there exists $r>0$ such that $V_{C_{r} \backslash D}\left(z_{0}\right)=1$. If $z_{0}$ is not $L$-regular we have

$$
\lim _{r \rightarrow 0^{+}} V_{C_{r} \backslash D}\left(z_{0}\right)=0 .
$$

ProOF. See, e.g., Lemma 1.3 in [L1], Theorem 7.2 in [B] and Theorem 4.3.1 in [Ba].

PROPOSITION 4.2. Let $\left(F_{k}\right)_{k \in \mathbf{N}}$ be a sequence of compact subsets of $\mathbf{R}^{n+1}$, and let $F$ be another compact set such that $F \subset \bigcup_{k=1}^{\infty} F_{k}$. Then

$$
V_{F} \leq \sum_{k=1}^{\infty} V_{F_{n}} .
$$

PROOF. It follows by a step-by-step imitation of the argument in the proof of Theorem 1.2 in [L1].

In stating the next result we recall the definition (1.8) of $L$-capacity.

PROPOSITION 4.3. There exists a constant $C$, depending only on $L$, such that for every compact $F \subset \mathbf{R}^{n+1}$

$$
\begin{aligned}
& C \operatorname{cap}_{H}(F) \leq \operatorname{cap}_{L}(F) \leq C^{-1} \operatorname{cap}_{H}(F), \\
& C \operatorname{cap}_{L^{*}}(F) \leq \operatorname{cap}_{L}(F) \leq C^{-1} \operatorname{cap}_{L^{*}}(F),
\end{aligned}
$$

where

$$
L^{*}=\operatorname{div}\left(A(x, t) \nabla_{x}\right)+D_{t}
$$

is the adjoint of $L$.

ProOF. By (2.4) and (1.8) we obtain

$$
C_{2} \operatorname{cap}_{H_{\alpha_{2}}}(F) \leq \operatorname{cap}_{L}(F) \leq C_{1} \operatorname{cap}_{H_{\alpha_{1}}}(F) .
$$

The proof of (4.9) now follows directly by Proposition 2 in [L2]. The proof of (4.10) follows by (4.9) and the fact that

$$
\operatorname{cap}_{H}(F)=\operatorname{cap}_{H^{*}}(F),
$$

where $H^{*}=\Delta+D_{t}$ (see [W2]).

LEMMA 4.2. $z_{0}$ is $L$-regular for $D$ iff for every $r, c>0$ it is $L$-regular for the open set $D_{r, c}$, where we have set

$$
D_{r, c}=D \cup\left\{z \in \stackrel{\circ}{C}_{r} \mid \Gamma\left(z_{0} ; z\right)<(4 \pi c)^{-n / 2}\right\} .
$$

ProOF. We observe preliminarily that $z_{0} \in \partial D_{r, c}$. Since $D_{r, c} \supset D$, it is clear that $z_{0}$ is $L$-regular for $D$ if it is $L$-regular for $D_{r, c}$, for any $r, c>0$. It is then enough to show that if $z_{0}$ is $L$-irregular for $D_{r, c}$ then it is $L$-irregular for $D$. Now if $z_{0}$ is $L$-irregular for $D_{r, c}$ we have

$$
\lim _{\rho \rightarrow 0^{+}} V_{C_{\rho} \backslash D_{r, c}}\left(z_{0}\right)=0 .
$$

But for $0<\rho<r$

$$
C_{\rho} \backslash D \subset\left(C_{\rho} \backslash D_{r, c}\right) \cup F_{\rho, c},
$$


where

$$
F_{\rho, c}=\left\{z \in C_{\rho} \mid \Gamma\left(z_{0}, z\right) \leq(4 \pi c)^{-n / 2}\right\}
$$

Hence

$$
V_{C_{\rho} \backslash D}\left(z_{0}\right) \leq V_{C_{\rho} \backslash D_{r, c}}\left(z_{0}\right)+V_{F_{\rho, c}}\left(z_{0}\right) .
$$

To prove that $z_{0}$ is $L$-irregular for $D$ by $(4.16)$ and (4.14) it is then s'ufficient to show that

$$
\lim _{\rho \rightarrow 0^{+}} V_{F_{\rho, c}}\left(z_{0}\right)=0 .
$$

To this purpose we observe that if $\mu$ is the equilibrium measure of $F_{\rho, c}$, we have

$$
\begin{aligned}
V_{F_{\rho, c}}\left(z_{0}\right) & =\int_{F_{\rho, c}} \Gamma\left(z_{0} ; \zeta\right) d \mu(\varsigma) \leq(4 \pi c)^{-n / 2} \mu\left(F_{\rho, c}\right) \\
& =(4 \pi c)^{-n / 2} \operatorname{cap}_{L}\left(F_{\rho, c}\right) \leq(4 \pi c)^{-n / 2} \operatorname{cap}_{L}\left(C_{\rho}\right) \leq(4 \pi c)^{-n / 2} \Lambda \rho^{n},
\end{aligned}
$$

where $\Lambda>0$ is a number which depends only on $L$. The last inequality is justified by noting that $\operatorname{cap}_{H}(\lambda F)=\lambda^{n} \operatorname{cap}_{H}(F)$ for any compact set $F$, where $\lambda F=$ $\left\{\left(\lambda x, \lambda^{2} t\right) /(x, t) \in F\right\}$, and by (4.9). By (4.18) the proof is complete.

We are now ready to give the

PROOF OF THEOREM 1.1. Necessity. This is the easy part of the proof. We show that if for any $\lambda \in(0,1)$

$$
\sum_{k=1}^{\infty} \lambda^{-k(n / 2)} \operatorname{cap}_{L}\left(D\left(\lambda^{k}\right)\right)<+\infty
$$

then $z_{0}$ is $L$-irregular for $D$. In (4.19) we have set

$$
\begin{aligned}
D\left(\lambda^{k}\right) & =D^{c} \cap A\left(z_{0} ; \lambda^{k}\right) \\
& =D^{c} \cap\left[\left\{z \in \mathbf{R}^{n+1} \mid\left(4 \pi \lambda^{k+1}\right)^{-n / 2} \geq \Gamma\left(z_{0} ; z\right) \geq\left(4 \pi \lambda^{k}\right)^{-n / 2}\right\} \cup\left\{z_{0}\right\}\right]
\end{aligned}
$$

(see (1.9)). By (4.19) there exists $p \in \mathbf{N}$ such that

$$
\sum_{k=p}^{\infty} \lambda^{-k(n / 2)} \operatorname{cap}_{L}\left(D\left(\lambda^{k}\right)\right)<\lambda^{n / 2} .
$$

Now if $c=\lambda^{p}$ we have $C_{r} \backslash D_{r, c} \subset \bigcup_{k=p}^{\infty} D\left(\lambda^{k}\right)$. Therefore, by (4.8) we obtain (4.21)

$$
V_{C_{r} \backslash D_{r, c}}\left(z_{0}\right) \leq \sum_{k=p}^{\infty} V_{D\left(\lambda^{k}\right)}\left(z_{0}\right) \leq \sum_{k=p}^{\infty} \operatorname{cap}_{L}\left(D\left(\lambda^{k}\right)\right)\left(4 \pi \lambda^{k+1}\right)^{-n / 2}<(4 \pi)^{-n / 2}<1 .
$$

By Proposition 4.1 we conclude that $z_{0}$ is $L$-irregular for $D_{r, c}$. By Proposition 4.1 it follows that $z_{0}$ is $L$-irregular for $D$.

Sufficiency. We assume that (1.10) holds. In what follows we will proceed along the lines of the proof of the sufficiency in [EG]. To begin with, since by (1.10) at least one of the four series $\sum_{k=1}^{\infty} \lambda^{-(4 k+i) n / 2} \operatorname{cap}_{L}\left(D\left(\lambda^{4 k+1}\right)\right), i=0,1,2,3$, diverges, we may assume that

$$
\sum_{k=1}^{\infty} \lambda^{-4 k n / 2} \operatorname{cap}_{L}\left(D\left(\lambda^{4 k}\right)\right)=+\infty
$$


Since $\operatorname{cap}_{L}\left(C_{r}\right) \rightarrow 0$ as $r \rightarrow 0^{+}$, for every $k \in \mathbf{N}$ we can choose $r_{k}>0$ such that replacing $D\left(\lambda^{4 k}\right)$ by $D\left(\lambda^{4 k}\right) \cap\left(\mathbf{R}^{n+1} \backslash C_{r_{k}}\right)=F_{4 k},(4.22)$ still holds. We will show that $z_{0}$ is $L$-regular for

$$
\tilde{D}=\stackrel{\circ}{C_{1}} \backslash\left(\bigcup_{k=1}^{\infty} F_{4 k} \cup\left\{z_{0}\right\}\right)=\stackrel{\circ}{C}_{1} \backslash F
$$

Since $D \cap \stackrel{\circ}{C}_{1} \subset \tilde{D}$, this will be enough to conclude that $z_{0}$ is $L$-regular for $D$. We denote by $V$ the equilibrium potential of $F$ and set

$$
W=1-V \text {. }
$$

Claim. $W(0)=0$.

It is clear that by Proposition 4.1 the claim implies the $L$-regularity of $z_{0}$. For any $\varepsilon>0$ and any $k \in \mathbf{N}$ we choose a compact set $F_{4 k}^{\varepsilon}$ such that

$$
\stackrel{\circ}{F_{4 k}^{\varepsilon}} \supset F_{4 k}, F_{4 k}^{\varepsilon} \subseteq F_{4 k}^{\varepsilon^{\prime}} \quad \text { if } 0<\varepsilon<\varepsilon^{\prime}, \quad \bigcap_{\varepsilon>0}\left(\bigcup_{k} F_{4 k}^{\varepsilon} \cup\left\{z_{0}\right\}\right)=F .
$$

Moreover, we request that

$$
F_{4 k}^{\varepsilon} \subset\left\{z \in \mathbf{R}^{n+1} \mid\left(4 \pi \lambda^{k+2}\right)^{-n / 2}>\Gamma\left(z_{0} ; z\right)>\left(4 \pi \lambda^{4 k-1}\right)^{-n / 2}\right\}
$$

and that $F^{\varepsilon}=\bigcup_{k} F_{4 k}^{\varepsilon} \cup\left\{z_{0}\right\}$ be compact. Let now $V^{\varepsilon}$ and $\mu^{\varepsilon}$ be respectively the equilibrium potential and the equilibrium measure of $F^{\varepsilon}$. From now on, to simplify the notation we drop the subscript $k$. We assume that $k \in \mathbf{N}$ has been fixed throughout the discussion and we simply write $V^{*}$ and $\nu^{*}$ to denote respectively the equilibrium potential and measure of $F_{4 k}$ with respect to the operator $L^{*}$ in (4.11). By Lemma 4.1 we can find two sequences $\left(V_{j}^{\varepsilon}\right)_{j \in \mathbf{N}}, V_{j}^{\varepsilon} \in P \cap C^{2}$, and $\left(V_{j}^{*}\right)_{j \in \mathbf{N}}, V_{j}^{*} \in P^{*} \cap C^{2}\left(P^{*}\right.$ is defined as in (4.6) replacing $L$ with its adjoint $\left.L^{*}\right)$, such that

$$
0 \leq V_{j}^{\varepsilon} \uparrow V^{\varepsilon}, \quad 0 \leq V_{j}^{*} \uparrow V^{*}
$$

for every $\delta>0 \exists p \in \mathbf{N}: V_{j}^{\varepsilon}(z)=V^{\varepsilon}(z)$ for $j \geq p$, for every $z \in \mathbf{R}^{n+1}$ such that $d\left(z ; F^{\varepsilon}\right) \geq \delta$; moreover, $V_{j}^{*}(z)=V^{*}(z)$ for $j \geq p$ and every $z \in \mathbf{R}^{n+1}$ such that $d\left(z ; F_{4 k}\right) \geq \delta$.

In particular, we have

$$
L^{*} V_{j}^{*}=-\nu_{j}^{*}
$$

where $\nu_{j}^{*} \in C\left(\mathbf{R}^{n+1}\right), \nu_{j}^{*} \geq 0$ and $\operatorname{supp} \nu_{j}^{*}=$ compact. By (4.27) we infer that for every Borel subset $A \subseteq \mathbf{R}^{n+1}$, such that $\stackrel{\circ}{\supset} F_{4 k}$

$$
\nu_{j}^{*}(A) \rightarrow \nu^{*}(A), \quad \text { as } j \rightarrow \infty,
$$

where obviously $\nu_{j}^{*}(A)=\int_{A} \nu_{j}^{*}(z) d z$. Moreover, as $V^{\varepsilon} \equiv 1$ on $\stackrel{\circ}{F}_{4 k}^{\varepsilon}$, it is not restrictive to suppose that

$$
V^{\varepsilon}(z)=1 \quad \text { for every } z \in \operatorname{supp} \nu_{j}^{*} .
$$


At this point we fix $\sigma>0$ as in the discussion following (2.7). We then choose a $\lambda \in(0,1)$ such that $\lambda \leq \frac{3}{8} \sigma$, and set $r=\frac{1}{2} \lambda^{4 k-2}$. With this choice $\lambda^{4 k-1}<\sigma r$, therefore (see $\S 2$ ) we have

$$
Q(2 r) \supset \Omega_{\sigma r} \supset \Omega_{\lambda^{4 k-1}} \supset F_{4 k}^{\varepsilon} .
$$

In $Q(2 r)$ we set $W_{j}^{\varepsilon} \equiv 1-V_{j}^{\varepsilon}$ and write

$$
W_{j}^{\varepsilon}=p_{j}^{\varepsilon}-s_{j}^{\varepsilon}
$$

where $p_{j}^{\varepsilon}$ solves the problem

$$
\left\{\begin{array}{l}
L p_{j}^{\varepsilon}=0 \text { in } Q(2 r) \\
p_{j}^{\varepsilon} \mid \partial Q(2 r) \backslash\left\{z_{0}\right\}=W_{j}^{\varepsilon}
\end{array}\right.
$$

and $s_{j}^{\varepsilon}=p_{j}^{\varepsilon}-W_{j}^{\varepsilon}$ (we remark that for $r$ sufficiently small every point of $\partial Q(2 r) \backslash\left\{z_{0}\right\}$ is $L$-regular for $Q(2 r)$; this has been proved in [GL]).

Following the argument in $[\mathbf{E G}]$ we have

$$
\begin{aligned}
&\left(\inf _{\Omega_{3 \sigma r / 4}} p_{j}^{\varepsilon}\right)^{2} \nu_{j}^{*}\left(\Omega_{3 \sigma r / 4}\right) \leq \int_{\Omega_{3 \sigma r / 4}}\left(p_{j}^{\varepsilon}\right)^{2}(z) \nu_{j}^{*}(z) d z \quad(\text { by }(4.32)) \\
& \leq \int_{Q(2 r)}\left(p_{j}^{\varepsilon}\right)^{2}(z) \nu_{j}^{*}(z) d z \quad(\text { by }(4.33) \text { and }(4.31)) \\
&=\int_{Q(2 r)}\left(s_{j}^{\varepsilon}\right)^{2}(z) \nu_{j}^{*}(z) d z=-\int_{Q(2 r)}\left(s_{j}^{\varepsilon}\right)^{2}(z) L^{*} V_{j}^{*}(z) d z \quad(\text { by }(4.29)) \\
&=-\int_{Q(2 r)} L\left(\left(s_{j}^{\varepsilon}\right)^{2}\right)(z) V_{j}^{*}(z) d z \quad\left(\text { recall } s_{j}^{\varepsilon}=0 \text { on } \partial Q(2 r) \backslash\left\{z_{0}\right\}\right) \\
&=-2 \int_{Q(2 r)} s_{j}^{\varepsilon}(z) L s_{j}^{\varepsilon}(z) V_{j}^{*}(z) d z-2 \int_{Q(2 r)} A(z) \nabla_{x} s_{j}^{\varepsilon}(z) \cdot \nabla_{x} s_{j}^{\varepsilon}(z) V_{j}^{*}(z) d z \\
& \leq-2 \int_{Q(2 r)} s_{j}^{\varepsilon}(z) L s_{j}^{\varepsilon}(z) V_{j}^{*}(z) d z \quad(\text { by }(4.33) \text { and }(4.34)) \\
&\left.=2 \int_{Q(2 r)} s_{j}^{\varepsilon}(z) L W_{j}^{\varepsilon}(z) V_{j}^{*}(z) d z \quad{ }^{\varepsilon}\right) \\
&\left(\operatorname{since} L W_{j}^{\varepsilon}=-L V_{j}^{\varepsilon} \geq 0,0 \leq s_{j}^{\varepsilon}=p_{j}^{\varepsilon}-W_{j}^{\varepsilon} \leq p_{j}^{\varepsilon} \leq 1, \text { and } 0 \leq V_{j}^{*} \leq 1\right) \\
& \leq 2 \int_{\Omega_{2 r}} L W_{j}^{\varepsilon}(z) d z .
\end{aligned}
$$

Now by Theorem 1.4 and Corollary 1.2 we obtain from (4.35)

$$
\left(f_{I_{r}} p_{j}^{\varepsilon}(x,-\eta r) d x\right)^{2} \nu_{j}^{*}\left(\Omega_{3 \sigma r / 4}\right) r^{-n / 2} \leq C_{n, \alpha}\left[W_{j, 2 \alpha^{2} r}^{\varepsilon}\left(z_{0}\right)-W_{j, 2 \alpha r}^{\varepsilon}\left(z_{0}\right)\right]
$$

where $\alpha>1$ has to be chosen. Now we take $\alpha=\lambda^{-1}$ so that $2 \alpha r=\lambda^{4 k-3}$, $2 \alpha^{2} r=\lambda^{4 k-4}$, hence

$$
\begin{aligned}
& W_{j, 2 \alpha^{2} r}^{\varepsilon}\left(z_{0}\right)-W_{j, 2 \alpha r}^{\varepsilon}\left(z_{0}\right)=W_{j, \lambda^{4 k-4}}^{\varepsilon}\left(z_{0}\right)-W_{j, \lambda}^{\varepsilon}{ }^{4 k-3} \\
& \leq \sum_{j=1}^{4}\left(Z_{j, \lambda^{4 k-i}}^{\varepsilon}\left(z_{0}\right)-W_{j, \lambda^{4 k-i+1}}^{\varepsilon}\left(z_{0}\right)\right) \quad \text { (by Corollary 1.2) }
\end{aligned}
$$


Now by (4.27) and (4.30) we have as $j \rightarrow \infty$ (4.38)

$$
W_{j, \rho}^{\varepsilon} \rightarrow W_{\rho}^{\varepsilon} \text { for } \rho>0, \quad \nu_{j}^{*}\left(\Omega_{3 \sigma r / 4}\right) \rightarrow \nu^{*}\left(\Omega_{3 \sigma r / 4}\right)=\operatorname{cap}_{L^{*}}\left(F_{4 k}\right) \quad(\text { see }(4.4)),
$$

since $F_{4 k} \subset \Omega_{3 \sigma r / 4}$ (we observe that $\lambda^{4 k}<3 \sigma r / 4$ ). Moreover, since $F \cap\{(x, t) \in$ $Q(2 r) \mid t \leq-\eta r\}=\varnothing$, we have $s_{j}^{\varepsilon}(x, t)=0$, for $j$ large enough, if $(x, t) \in Q_{2 r}$ and $t \leq-\eta r$. By (4.38), taking into account (4.37), and letting $j \rightarrow \infty$ we obtain

$$
\begin{gathered}
\left(f_{|x|^{2} \leq R_{r}^{1}(-\eta r)} W^{\varepsilon}(x,-\eta r) d x\right)^{2} \operatorname{cap}_{L^{*}}\left(F_{4 k}\right) r^{-n / 2} \\
\leq c_{n, \lambda} \sum_{i=1}^{4}\left[W_{\lambda^{4 k-i}}^{\varepsilon}\left(z_{0}\right)-W_{\lambda^{4 k-i+1}}^{\varepsilon}\left(z_{0}\right)\right] .
\end{gathered}
$$

Now as $\varepsilon \downarrow 0 W^{\varepsilon} \uparrow \tilde{W}$, where $0 \leq \tilde{W} \leq 1$ and $\tilde{W}=1-V$ in an open neighborhood of the set $\left\{\left.(x,-\eta r)|| x\right|^{2} \leq R_{r}^{1}(-\eta r)\right\}$. The proof of this fact follows exactly as in the proof of (5.6) in [EG]. Therefore, letting $\varepsilon \rightarrow 0^{+}$in (4.39) we finally obtain

$$
\begin{gathered}
\left(f_{|x|^{2} \leq R_{r}^{1}(-\eta r)} W(x,-\eta r) d x\right)^{2} \operatorname{cap}_{L^{*}}\left(F_{4 k}\right) r^{-n / 2} \\
\leq c_{n, \lambda} \sum_{i=1}^{4}\left[\tilde{W}_{\lambda^{4 k-i}}\left(z_{0}\right)-\tilde{W}_{\lambda^{4 k-i+1}}\left(z_{0}\right)\right]
\end{gathered}
$$

Recalling now that $r=(1 / 2) \lambda^{4 k-2}$, and setting

$$
\beta_{k}=f_{|x|^{2} \leq R_{r}^{1}\left(-1 / 2 \eta \lambda^{4 k-2}\right)} W\left(x,-1 / 2 \eta \lambda^{4 k-2}\right) d x,
$$

by $(4.10),(4.40)$ and the fact that $\left.\sum_{k=1}^{\infty} \sum_{i=1}^{4}\left[\tilde{W}_{\lambda^{4 k-i}}\left(z_{0}\right)-\tilde{W}_{\lambda^{4 k-i+1}}\left(z_{0}\right)\right]\right)$ is a telescopic series, we conclude that

$$
\sum_{k=1}^{\infty} \beta_{k}^{2} \operatorname{cap}_{L}\left(F_{4 k}\right)\left(\lambda^{4 k}\right)^{-n / 2}<+\infty
$$

By (4.22) we must have

$$
\varliminf_{k \rightarrow \infty} \beta_{k}=0 .
$$

With (4.42) in hand the rest of the proof of the claim $(W(0)=0)$ follows exactly as in the case of the heat equation. We leave out the details referring the reader to [EG].

Our next task is to prove Theorem 1.2. We do this by first presenting the

PROOF OF THEOREM 1.8. We prove that if $z_{0}=\left(x_{0}, t_{0}\right)=(0,0) \in \partial D$ is not $M$-regular, then it is not $L$-regular. In the sequel since we want to distinguish between the two operators $L$ and $M$, we will explicitly indicate dependence on one or the other. Since by Lemma $4.2 z_{0}$ is $L$-irregular for $D$ iff it is $L$-irregular for $\tilde{D}=D \cup\left\{z \in \stackrel{\circ}{C}_{r} \mid \Gamma_{L}\left(z_{0} ; z\right)<(4 \pi c)^{-n / 2}\right\}$, we may assume that $C_{r} \backslash D \subset\{z \in$ $\left.\mathbf{R}^{n+1} \mid \Gamma_{L}\left(z_{0} ; z\right) \geq(4 \pi c)^{-n / 2}\right\}$. Hence by (1.44) we have

$$
\Gamma_{L}\left(z_{0} ; z\right) \leq B \Gamma_{M}\left(z_{0} ; z\right) \text { for } z \in C_{r} \backslash D .
$$


If $z_{0}$ is $M$-irregular, then by Theorem 1.1

$$
\sum_{k=1}^{\infty} \lambda^{-k n / 2} \operatorname{cap}_{M}\left(D^{m}\left(\lambda^{k}\right)\right)<+\infty,
$$

for some $\lambda \in(0,1)$. (4.44) implies that for every $\varepsilon>0$ there exists $p \in \mathbf{N}$ such that

$$
\sum_{k=p}^{\infty} \lambda^{-k n / 2} \operatorname{cap}_{M}\left(D^{M}\left(\lambda^{k}\right)\right)<\varepsilon .
$$

Now, as in the proof of the necessity in Theorem 1.1 we take $c=\lambda^{p}$ and we assume that $p$ is such that $\lambda^{p} \leq r$. We have

$$
C_{r} \backslash D_{r, c}^{M} \subset \bigcup_{k=p}^{\infty} D^{M}\left(\lambda^{k}\right),
$$

and therefore as in (4.21) we obtain

$$
V_{C_{r} \backslash D_{r, c}^{M}}^{L}\left(z_{0}\right) \leq \sum_{k=p}^{\infty} V_{D^{M}\left(\lambda^{k}\right)}^{L}\left(z_{0}\right) .
$$

Now, if $\mu_{k}$ is the $L$-measure of equilibrium of $D^{M}\left(\lambda^{k}\right)$ we have by (4.43)

$$
\begin{aligned}
V_{D^{M}\left(\lambda^{k}\right)}^{L}\left(z_{0}\right) & =\int_{\mathbf{R}^{n+1}} \Gamma_{L}\left(z_{0} ; \varsigma\right) d \mu_{k}(\varsigma) \leq B \int_{\mathbf{R}^{n+1}} \Gamma_{M}\left(z_{0} ; \varsigma\right) d \mu_{k}(\varsigma) \\
& \leq C^{\prime} \lambda^{-k n / 2} \mu_{k}\left(\mathbf{R}^{n+1}\right)=C^{\prime} \lambda^{-k n / 2} \operatorname{cap}_{L}\left(D^{M}\left(\lambda^{k}\right)\right) \\
& \leq C^{\prime \prime} \lambda^{-k n / 2} \operatorname{cap}_{M}\left(D^{M}\left(\lambda^{k}\right)\right) \quad(\text { by }(4.9)),
\end{aligned}
$$

where $C^{\prime \prime}$ is a constant that does not depend on $\varepsilon$. (4.47), (4.48) and (4.45) can be summarized saying that for every $\varepsilon>0$

$$
V_{C_{r} \backslash D_{r, c}^{M}}^{L}\left(z_{0}\right) \leq C^{\prime \prime} \varepsilon .
$$

On the other hand (1.44) implies that

$$
C_{r} \backslash D_{r, B^{-2 / n} c}^{L} \subset C_{r} \backslash D_{r, c}^{M},
$$

where $B$ is as in (1.44). If we take $C^{\prime \prime} \varepsilon<1$ in (4.49), (4.50) gives

$$
V_{C_{r} \backslash D_{r, B}^{L-2 / n_{c}}}^{L}\left(z_{0}\right)<1 .
$$

Lemma 4.2 and Proposition 4.1 allow us to conclude that $z_{0}$ is $L$-irregular.

We are now in a position to give the

PROOF OF THEOREM 1.2. As before, we suppose that $z_{0} \in \partial D$ is the origin. Moreover, as this is not restrictive, we can suppose that

$$
a_{i j}\left(z_{0}\right)=b_{i j}\left(z_{0}\right)=\delta_{i j}, \quad i, j=1, \ldots, n .
$$

The precise estimates of the fundamental solutions $\Gamma_{L}$ and $\Gamma_{M}$ (see Theorem 1.3) allow us to

Claim. There exists a constant $B>0$ such that for an $r$ sufficiently small,

$$
\Gamma_{L}\left(z_{0} ; z\right) \leq B \Gamma_{M}\left(z_{0} ; z\right)
$$

for every $z$ for which $\Gamma_{L}\left(z_{0} ; z\right)>(4 \pi r)^{-n / 2}$. 
Let us assume for a moment that the claim is true. By Theorem 1.8 we conclude that if $z_{0}$ is $L$-regular, then $z_{0}$ is $M$-regular. Reversing the roles of $L$ and $M$ in the claim would complete the proof.

The proof of the claim is based on (1.18). As for the first equation in (2.15) for $k \in \mathbf{N}$ large $(k>n / 2$ will suffice $)$ we can write

$$
\Gamma_{L}\left(z_{0} ; z\right)=G_{L}\left(z_{0} ; z\right) \eta_{k}^{L}(z)+w_{k}^{L}(z)
$$

for $z \in C_{r}$ (see (2.10)) and $r$ sufficiently small. In (4.54)

$$
G_{L}\left(z_{0}, z\right)=(-4 \pi t)^{-n / 2} \exp \left(\frac{d_{L}^{2}(0, x,-t)}{4 t}\right)
$$

(remember, $t<0$ now!), $\eta_{k}^{L}$ is defined as in (2.18), and $w_{k}^{L}(z)=o(1)$ as $z \rightarrow z_{0}$. As analogous expansion holds for $\Gamma_{M}\left(z_{0} ; z\right)$. On the other hand, taking (4.52) into account, denoting by $\exp _{t}^{L}$ the exponential map with pole $x_{0}=0 \in \mathbf{R}^{n}$ in the $L$-metric $g_{i j}(t)=a^{i j}(t)$, we can write

$$
d_{L}^{2}(x, 0,-t)=\left|\left(\exp _{t}^{L}\right)^{-1}(x)\right|^{2}=\left|\left(\exp _{0}^{L}\right)^{-1}(x)+O(t x)\right|=|x|^{2}\left(1+\varphi_{L}(x, t)\right)
$$

where $\varphi_{L}(x, t)=O(|x|+|t|)$ for $(x, t) \rightarrow z_{0}$.

Analogously, we have

$$
d_{M}^{2}(x, 0,-t)=|x|^{2}\left(1+\varphi_{M}(x, t)\right),
$$

where $\varphi_{M}(x, t)=O(|x|+|t|)$ as $(x, t) \rightarrow z_{0}$.

The claim now follows directly by (4.55), (4.56), (4.54) and its analog for $\Gamma_{M}$, and the fact that on the set where $\Gamma_{L}\left(z_{0} ; z\right)$ is large, namely where $\Gamma_{L}\left(z_{0} ; z\right) \geq$ $(4 \pi r)^{-n / 2}$ we have

$$
|x|^{2}=O(|t| \ln |t|) \quad \text { as } t \rightarrow 0 .
$$

ACKNOWLEDGment. The first author wishes to thank the School of Mathematics of the University of Minnesota for the kind hospitality during the final draft of this work.

\section{REFERENCES}

[A] D. G. Aronson, Non negative solutions of linear parabolic equations, Ann. Norm. Sup. Pisa 22 (1968), 607-694.

[Ba] H. Bauer, Harmonische Räume und ihre Potentialtheorie, Lecture Notes in Math., vol. 22, Springer-Verlag, 1966.

[BGM] M. Berger, P. Gauduchon and E. Mazet, Le spectre d'une variété Riemanniene, Lecture Notes in Math., vol. 194, Springer-Verlag, 1971.

[B] M. Brelot, On topologies and boundaries in potential theory, Lecture Notes in Math., vol. 175, Springer-Verlag, 1971.

[EG] L. C. Evans and R. F. Gariepy, Wiener's criterion for the heat equation, Arch. Rational Mech. Anal. 78 (1982), 293-314.

[FG] E. B. Fabes and N. Garofalo, Mean value properties of solutions to parabolic equations with variable coefficients, J. Math. Anal. Appl. 121 (1987), 305-316.

[Fe] H. Federer, Geometric measure theory, Springer-Verlag, 1969.

[Fr] A. Friedman, Partial differential equations of parabolic type, Prentice-Hall, Englewood Cliffs, N. J., 1964.

[Fu] W. Fulks, A mean value theorem for the heat equation, Proc. Amer. Math. Soc. 17 (1966), 6-11. 
[GL] N. Garofalo and E. Lanconelli, Asymptotic behavior of fundamental solutions and potential theory of parabolic operators with variable coefficients, preprint.

[GZ] R. F. Gariepy and W. P. Ziemer, Thermal capacity and boundary regularity, J. Differential Equations 45 (1982), 374-388.

[K] Y. Kannai, Off diagonal short time asymptotics for fundamental solutions of diffusion equations, Comm. 2 (1977), 781-830.

[L1] E. Lanconelli, Sul problema di Dirichlet per l'equazione del calore, Ann. Mat. Pura Appl. 97 (1973), 83-114.

[L2] __ Sul problema di Dirichlet per equazioni paraboliche del secondo ordine a coefficienti discontinui, Ann. Mat. Pura Appl. 106 (1975), 11-37.

[L3] _ Sul confronto della regolarità dei punti di frontiera rispetto ad operatori lineari parabolici diversi, Ann. Mat. Pura Appl. 114 (1977), 207-227.

[La] E. M. Landis, Necessary and sufficient conditions for regularity of a boundary point in the Dirichlet problem for the heat-conduction equation, Soviet Math. 10 (1969), 380-384.

[LSW] W. Littman, G. Stampacchia and H. Weinberger, Regular points for elliptic equations with discontinuous coefficients, Ann. Scuola Norm. Sup. Pisa 17 (1963), 45-79.

[N] A. A. Nohruzov, On some regularity criteria for boundary points for linear and quasi-linear parabolic equations, Dokl. Akad. Nauk SSSR 209 (1973). (Russian)

[Pe] I. Petrovsky, Zur ersten Randwertaufgabe der Warmeleitungsgleichung, Compositio Math. 1 (1935), 383-419.

[P1] B. Pini, Sulle equazioni a derivate parziali lineari del secondo ordine in due variabili di tipo parabolico, Ann. Mat. Pura Appl. 32 (1951), 179-204.

[P2] _ Maggioranti e minoranti delle soluzioni delle equazioni paraboliche, Ann. Mat. Pura Appl. 37 (1954), 249-264.

[P3] __ Sulla soluzione generalizzata di Wiener per il primo problema di valori al contorno nel caso parabolico, Rend. Sem. Mat. Univ. Padova 23 (1954), 422-434.

[Wa1] N. A. Watson, A theory of temperatures in several variables, Proc. London Math. Soc. (3) 26 (1973), 385-417.

[Wa2] — Thermal capacity, Proc. London Math. Soc. 37 (1978), 342-362.

[W] N. Wiener, The Dirichlet problem, J. Math. Phys. 3 (1924), 127-146.

[Z] W. P. Ziemer, Behavior at the boundary of solutions of quasi-linear parabolic equations, J. Differential Equations 35 (1980), 291-305.

Dipaktimento di Matematica, University of Bologna, Bologna, Italy 